\title{
Robust Daily Exchange Rate Forecasting
}

by

Mark Blanchette, B.Math.

\begin{abstract}
A thesis submitted to the
Faculty of Graduate Studies and Research

in partial fulfillment of the requirements for the degree of
\end{abstract}

Master of Arts in Economics

Department of Economics

Carleton University

Ottawa, Ontario, Canada

C Copyright 2007, Mark Blanchette 


$\begin{array}{ll}\begin{array}{l}\text { Library and } \\ \text { Archives Canada }\end{array} & \begin{array}{l}\text { Bibliothèque et } \\ \text { Archives Canada }\end{array} \\ \begin{array}{l}\text { Published Heritage } \\ \text { Branch }\end{array} & \begin{array}{l}\text { Direction du } \\ \text { Patrimoine de l'édition }\end{array} \\ \begin{array}{l}\text { 395 Wellington Street } \\ \text { Ottawa ON K1A ON4 }\end{array} & \begin{array}{l}\text { 395, rue Wellington } \\ \text { Ottawa ON K1A ON4 } \\ \text { Canada }\end{array}\end{array}$

Your file Votre référence ISBN: 978-0-494-33738-7 Our file Notre référence ISBN: 978-0-494-33738-7

NOTICE:

The author has granted a nonexclusive license allowing Library and Archives Canada to reproduce, publish, archive, preserve, conserve, communicate to the public by telecommunication or on the Internet, loan, distribute and sell theses worldwide, for commercial or noncommercial purposes, in microform, paper, electronic and/or any other formats.

The author retains copyright ownership and moral rights in this thesis. Neither the thesis nor substantial extracts from it may be printed or otherwise reproduced without the author's permission.
AVIS:

L'auteur a accordé une licence non exclusive permettant à la Bibliothèque et Archives Canada de reproduire, publier, archiver, sauvegarder, conserver, transmettre au public par télécommunication ou par l'Internet, prêter, distribuer et vendre des thèses partout dans le monde, à des fins commerciales ou autres, sur support microforme, papier, électronique et/ou autres formats.

L'auteur conserve la propriété du droit d'auteur et des droits moraux qui protège cette thèse. $\mathrm{Ni}$ la thèse ni des extraits substantiels de celle-ci ne doivent être imprimés ou autrement reproduits sans son autorisation.
In compliance with the Canadian

Privacy Act some supporting forms may have been removed from this thesis.

While these forms may be included in the document page count, their removal does not represent any loss of content from the thesis.
Conformément à la loi canadienne sur la protection de la vie privée, quelques formulaires secondaires ont été enlevés de cette thèse.

Bien que ces formulaires aient inclus dans la pagination, il n'y aura aucun contenu manquant.

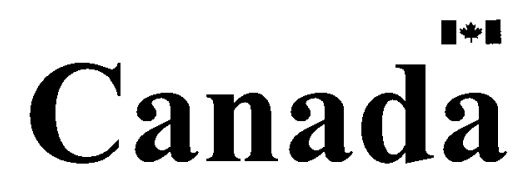




\begin{abstract}
Using a 15-year sample of eight daily foreign exchange rates, I develop robust methods for producing one-day-ahead out-of-sample forecasts. In particular, I show that the least absolute deviations (LAD) estimator can provide substantially better forecasts due to its robustness to outliers. I extend the Krasker-Welsch (1982) bounded influence methods to the LAD estimator to develop a two-stage estimation procedure, which I denote LAD-BI. I then develop a nonparametric data-driven estimation sample selection procedure, designed to select the optimal estimation sample in the presence of structural breaks. I show that the gross annual returns produced by forecasts from the LAD-BI estimator can be statistically significant, display evidence of market timing, and remain large in the presence of realistic transactions costs. Finally, I consider forecast combinations using a variety of weighting schemes in an attempt to further reduce parameter uncertainty. Overall, I find substantial evidence of inefficiencies in major foreign exchange markets.
\end{abstract}




\section{Table of Contents}

Abstract $\quad$ ii

Table of Contents

List of Tables iv

List of Appendices

1. Introduction 1

$\begin{array}{lll}\text { 2. } & \text { Forecasting Methodology and Data } & 7\end{array}$

3. Models and Estimators 11

4. LAD Estimation with Bounded Influence 13

5. Estimation Sample Selection 16

6. Evaluation of Out-of-Sample Forecasts 21

$\begin{array}{ll}\text { 7. Forecasting Results } & 24\end{array}$

8. Statistical Significance 28

9. Profitability and Risk 33

10. Forecast Combinations 41

$\begin{array}{ll}\text { 11. Conclusions } & 47\end{array}$

$\begin{array}{lr}\text { Appendices } & 50\end{array}$

References $\quad 56$

$\begin{array}{ll}\text { Tables } & 65\end{array}$

iii 


\section{List of Tables}

Table 1.1 Summary statistics, first differenced nominal exchange rate $\left(\Delta s_{t}\right)$

Table 1.2 Summary statistics, first differenced natural log nominal exchange rate $\left(\Delta l s_{t}\right)$

Table 2 Benchmark forecasts

Table 3.1.1 Forecasts based on rolling window, first differenced nominal exchange rate $\left(\Delta s_{t}\right)$, window size of $n_{T}=100$

Table 3.1.2 Forecasts based on rolling window, first differenced nominal exchange rate $\left(\Delta s_{t}\right)$, window size of $n_{T}=500$

Table 3.1.3 Forecasts based on rolling window, first differenced nominal exchange rate $\left(\Delta s_{t}\right)$, window size of $n_{T}=1000$

Table 3.2.1 Forecasts based on rolling window, first differenced natural log nominal exchange rate $\left(\Delta l s_{t}\right)$, window size of $n_{T}=100$

Table 3.2.2 Forecasts based on rolling window, first differenced natural log nominal exchange rate $\left(\Delta l s_{t}\right)$, window size of $n_{T}=500$

Table 3.2.3 Forecasts based on rolling window, first differenced natural log nominal exchange rate $\left(\Delta l s_{t}\right)$, window size of $n_{T}=1000$

Table 4.1 Forecasts based on expanding window, first differenced nominal exchange rate $\left(\Delta s_{t}\right)$

Table 4.2 Forecasts based on expanding window, first differenced natural log nominal exchange rate $\left(\Delta l s_{t}\right)$

Table 5.1.1 Forecasts based on optimal samples, first differenced nominal exchange rate $\left(\Delta s_{t}\right)$, sample optimization based on MSE

Table 5.1.2 Forecasts based on optimal samples, first differenced nominal exchange rate $\left(\Delta s_{t}\right)$, sample optimization based on MAE

Table 5.2.1 Forecasts based on optimal samples, first differenced natural log nominal exchange rate $\left(\Delta l s_{t}\right)$, sample optimization based on MSE 
Table 5.2.2 Forecasts based on optimal samples, first differenced natural log nominal exchange rate $\left(\Delta l s_{t}\right)$, sample optimization based on MAE

Table 6.1 Forecasts based on LAD-BI estimates, first differenced nominal exchange rate $\left(\Delta s_{t}\right)$, sample optimization based on MSE

Table 6.2 Forecasts based on LAD-BI estimates, first differenced nominal exchange rate $\left(\Delta s_{t}\right)$, sample optimization based on MAE

Table 7.1 Statistical significance, LAD estimates, with sample optimization based on MAE

Table 7.2 Statistical significance, LAD-BI estimates, with sample optimization based on MAE

Table 8.1 Profitability and risk, LAD-BI KTC estimates, with sample optimization based on MAE, no transactions costs

Table 8.2 Profitability and risk, LAD-BI KTC estimates, with sample optimization based on MAE, 0.5 bp one-way transactions cost

Table 8.3 Profitability and risk, LAD-BI KTC estimates, with sample optimization based on MAE, 1.0 bp one-way transactions cost

Table 8.4 Profitability and risk, LAD-BI KTC estimates, with sample optimization based on MAE, 2.0 bp one-way transactions cost

Table 9.1 Combination of forecasts across estimators, LAD-BI + MLE-t, sample optimization based on MAE

Table 9.2 Combination of forecasts across estimators, LAD-BI + MLE- $t+$ OLS, sample optimization based on MAE

Table 10.1 Combination of forecasts across estimation samples, RW500+ RW1000

Table 10.2 Combination of forecasts across estimation samples, RW100+ RW500 + RW1000

Table 10.3 Combination of forecasts across estimation samples, OWMSE + 92 OWMAE 
Table 10.4 Combination of forecasts across estimation samples, RW1000+ EW

Table 10.5 Combination of forecasts across estimation samples, EW + 94 OWMAE

Table 10.6 Combination of forecasts across estimation samples, RW1000+ 95 EW + OWMAE 


\section{List of Appendices}

$\begin{array}{lr}\text { Appendix A: Currency Codes } & 50\end{array}$

$\begin{array}{ll}\text { Appendix B: Data } & 50\end{array}$

Appendix C: Likelihood Functions 51

Appendix D: Bootstrap Estimation of Krasker-Welsch Tuning Constant 52

Appendix E: Bootstrapping of Diebold-Mariano Statistics 54

vii 


\section{Introduction}

Since Meese \& Rogoff (1983), the inability of economic theory to predict future exchange rates has been a puzzling issue. There have been some minor successes with structural fundamentals-based models at horizons of longer than one year, i.e. Mark (1995) and Chinn \& Meese (1995), but even these results have been called into question by Kilian (1999), Berkowitz \& Giorgianni (2001), and Faust, Rogers \& Wright (2003). Cheung, Chinn \& Pascual (2005) give a summary of the inconsistent and rather lackluster performance of empirical models based on fundamentals that were developed over the nineties. In more recent work, Mark \& Sul (2001) use panel cointegration techniques and find out-of-sample predictability for certain currency pairs at horizons of one and sixteen quarters. Kilian \& Taylor (2003) find evidence of predictability at horizons of two years and longer but not at shorter horizons.

In contrast to this, several authors have been able to show that the so-called technical analysis rules ${ }^{1}$ commonly utilized by foreign exchange traders have been historically profitable at short horizons (see Sweeny (1986), Levich \& Thomas (1993), Chang \& Osler (1999), LeBaron (1999), and Okunev \& White (2003), for example). These profits are suggestive of historical market inefficiencies. Technical analysis rules that are frequently used are typically either moving-average or filter rules, and are applied

\footnotetext{
${ }^{1}$ For a general introduction to common technical analysis rules, see Neely (1997). It is important to note that some authors refer to "technical analysis" as being synonymous with "chartist techniques," however the two methods are fundamentally different. Technical analysis rules provide a mechanical trading strategy that requires no intervention on the part of the trader, whereas chartist methods rely on the trader's own perceptions and experience in order to make forecasts.
} 
to weekly or higher frequency data. As Neely (1997) points out, technical analysis is used more than any other trading strategy by foreign exchange traders. Osler (2004) and Qi \& Wu (2006) find that profits from technical analysis rules have been significantly reduced in recent years, suggesting that market inefficiencies may have disappeared. However, there does not appear to be a consensus on this in the literature, as results from Okunev \& White (2003) and others are clearly contradictory. Interestingly, Okunev \& White (2003) identify an underlying autocorrelation structure of exchange rate return series as a primary factor in producing excess returns from technical analysis.

Several authors have attempted to determine if technical analysis excess returns can be explained by time series models. Taylor $(1992,1994)$ shows that some technical analysis rules are superior to ARIMA time series models. LeBaron (1992) argues that linear models are sufficient to explain technical analysis rules. Dewachter (2001) shows that a Markov switching model can explain the profitability of a moving average rule (specifically, an MA(30) rule), through its ability to capture the same returns structure in the model. Similarly, Dueker \& Neely (2007) combine trading signals from a Markov switching model with those from technical analysis rules to obtain returns that are slightly larger than those produced by technical analysis alone.

Some authors, including Neely, Weller \& Dittmar (1997) and Gençay (1999), have argued that technical analysis trading rules are able to capture nonlinearities in exchange rate returns. This argument appears to be supported by the positive results of Dewachter (2001) and Dueker \& Neely (2007) who use Markov switching models. In fact, there is significant evidence in the literature that there are important nonlinearities in exchange 
rates. In particular, Baillie \& Bollerslev (1989), Hsieh (1989), Baillie \& Bollerslev (1991), and Baillie, Bollerslev \& Mikkelsen (1996) find significant evidence of generalized autoregressive conditional heteroskedasticity (GARCH) effects in high frequency series. Brooks (1996) applies a large battery of nonlinearity tests and finds that GARCH dominates over other forms of nonlinearity. In a somewhat different approach, Kräger \& Kugler (1993) show that self-exciting threshold autoregressive (SETAR) models fit exchange rate series well in sample.

Though significant nonlinearities are found, rejection of linear models in sample does not imply better out-of-sample forecasting by nonlinear models, as Diebold \& Nason (1990) note. As such, several authors have attempted to evaluate the predictive ability of nonlinear models at high frequencies. Diebold \& Nason (1990) use a nonparametric highly-nonlinear locally weighted regression approach to forecast weekly exchange rates but are unable to improve on the random walk model in out-of-sample forecasting. Similarly, Meese \& Rose (1991) attempt to account for nonlinearities in structural models using monthly data, but cannot beat the random walk out of sample. Brooks (1997) evaluates a number of linear and nonlinear models (including GARCH, SETAR, and neural network models) using daily data and finds that none of the models display evidence of market timing ability. Clements \& Smith (2001) extend the work of Kräger \& Kugler (1993) and show that SETAR models can provide better forecasts than a random walk in simulated data, but this is dependent on how we measure forecast performance out of sample, and whether we condition on the current regime. Boero \& Marrocu (2002) evaluate SETAR, GARCH, and other nonlinear models using weekly 
data and show that out-of-sample forecast performance measured by mean squared error favours linear models. They further demonstrate, following Clements \& Smith (2001), that nonlinear models out-perform linear models only when conditioning on ex-post regime information. Meade (2002) examines frequencies of less than one day and shows that both parametric and nonparametric nonlinear models have some success, provided there are no transactions costs. However, following Diebold \& Nason (1990), he shows that there is little evidence supporting nonlinear models over linear ones at daily or lower frequencies.

Overall, there has been only been limited success with high frequency time series models thus far, including Nag \& Mitra (2002), Meade (2002), Sarantis (2006), and Dueker \& Neely (2007). We have already outlined the positive results of Meade (2002) and Dueker \& Neely (2007) above. Nag \& Mitra (2002) use artificial neural networks with daily exchange rates, and find that they perform well as measured by mean squared prediction error. Sarantis (2006) applies a time-varying parameters Bayesian VAR model to several daily frequency exchange rate series and finds statistically significant positive returns.

In this paper, we explore the possibility of market inefficiencies for eight daily exchange rates ${ }^{2}$ of six major currencies: British Pound Sterling, U.S. Dollar, Euro, Japanese Yen, Swiss Franc and Canadian Dollar. We attempt to capture inefficiencies

\footnotetext{
${ }^{2}$ Throughout this paper, we denote exchange rate pairs using ISO 4217 international banking codes. A list of currencies and their ISO 4217 codes that are used in this paper can be found in Appendix A. Currency pairs appear in the usual "base/quote" format. For example, the EURUSD pair denotes the number of U.S. dollars (the quoted currency) that can be exchanged for one Euro (the base currency).
} 
using data-driven methodology and simple univariate time series models. Unlike many forecasting papers in the literature, the focus of this paper will not be on modeling, but instead on improving forecasts using alternative empirical techniques. In particular, we will explore forecasting methodology that is robust to both extreme data points (outliers) and structural breaks in the time series.

As discussed above, there is no conclusive evidence that nonlinear models can outperform linear models in producing accurate high-frequency out-of-sample forecasts. Consequently, we use a simple first-order linear autoregressive model. As Brooks (1997) notes, complex models with many parameters may have a tendency to fit sample-specific noise in addition to the underlying process, which can cause poor out-of-sample performance. It is important to note that we are not arguing that there are no nonlinearities in exchange rate series. On the contrary, we believe that it has been decisively shown by a number of authors (mentioned above) that significant nonlinearities do exist in exchange rates. We are simply following the general conclusion of the literature that nonlinear models do not improve upon linear models in out-ofsample forecasts, and choose to take the more simple linear modeling path.

Model parameter uncertainty can be a major factor in attempting to produce accurate forecasts out of sample. Boothe \& Glassman (1987b) and Hsieh (1988) find significant evidence of leptokurtosis in exchange rates. This suggests the need to use estimators that are robust to heavy-tailed error distributions. Consequently, we apply the least absolute deviations (LAD) estimator to produce estimates of model parameters, with the goal of reducing parameter bias due to outliers. We also attempt to improve on the 
LAD estimator by using data-driven bounded influence techniques developed by Krasker $\&$ Welsch (1982) to remove noise from the time series.

Hsieh (1988) also indicates that exchange rate distributions appear to vary over time. Furthermore, the positive results from the use of Markov switching models (Dewachter, 2001 and Dueker \& Neely, 2007) suggest that subtle structural breaks in exchange rate series likely exist. Structural breaks relate directly to the predictability of a time series, as shown by Pesaran \& Timmermann (2005 \& 2007). In light of the importance of breaks in the present application, we review the usual rolling estimation window and expanding estimation window approaches, and suggest a new nonparametric data-driven estimation sample selection procedure.

We show that the market inefficiencies that we find in the form of excess returns are statistically significant using a modified Diebold-Mariano (1995) test, and that our forecasts display market timing ability as measured by the Pesaran-Timmermann (1992) test. We also demonstrate that these excess returns remain quite large in the presence of realistic transactions costs, and that the volatility-normalized returns are quite large relative to those of a major stock index.

Finally, we explore the possibility of using forecast combinations to further reduce parameter uncertainty. In particular, we examine combinations across forecasts from different estimators, and combinations across forecasts based on alternative estimation samples.

The remainder of this paper will proceed as follows. Section 2 will outline the general forecasting methodology, notation, and data that we use throughout this paper. 
Section 3 will introduce the models and estimators that we consider. In section 4 , we apply bounded influence methods to the LAD estimator to further improve robustness. Section 5 examines the estimation sample selection problem in the context of possibly statistically insignificant structural breaks. Section 6 defines the forecast accuracy statistics that we use. In section 7, we present forecasting results for the estimators and estimation samples under consideration. Section 8 explores the statistical significance of the results, and section 9 examines the profitability and risk of utilizing forecasts produced using our methodology. Section 10 considers the possibility of improved forecasts by applying forecast combination methods. Lastly, section 11 summarizes our findings and concludes.

\section{Forecasting Methodology and Data}

We are interested in producing one-step-ahead out-of-sample forecasts of eight exchange rate pairs using a 15-year sample of historical daily quotes. We consider five major currency pairs: Pound Sterling/U.S. Dollar (GBPUSD), U.S. Dollar/Swiss Franc (USDCHF), U.S. Dollar/Japanese Yen (USDJPY), U.S. Dollar/Canadian Dollar (USDCAD), and Euro/U.S. Dollar (EURUSD). We also consider three Euro cross pairs: Euro/Swiss Franc (EURCHF), Euro/Japanese Yen (EURJPY), and Euro/Pound Sterling (EURGBP). This gives us a combination of high volume majors and lower volume crosses that will allow us to evaluate our methods for consistency across currency pairs. The variety of currencies that we evaluate is also potentially significant as it will allow us 
to assess the performance of our forecasting methodology over a spectrum of series with possibly quite different dynamics.

We obtained daily frequency exchange rate series for each of the eight currency pairs that cover a relatively recent time period. The GBPUSD, USDCHF, USDJPY, and USDCAD series cover January 2, 1991 to October 25, 2006, resulting in a sample of 4085 observations. The EURUSD, EURCHF, EURJPY, and EURGBP series cover January 4, 1999 to October 25, 2006, resulting in a sample of 2019 observations for these pairs. Further technical details about the data used in this paper can be found in Appendix B.

There are two qualitative points of note regarding the data that we use. First, we use samples that start no earlier than January 1991. By this time, markets for the currencies that we consider were extremely liquid ${ }^{3}$ and trading infrastructure was highly advanced. Also, over this time period we saw the rise of widespread information technology and the prevalence of 24 -hour news coverage. Consequently, we believe that barriers to market efficiency, such as slow propagation of news events and large transactions costs, should have been very minimal over this time period.

Second, it is also important to note that the exchange rate series that we use consist of actual historical quoted rates. As such, these are the same rates that were available to traders active in the market at the time under consideration. These rates are superior to

\footnotetext{
${ }^{3}$ The Bank for International Settlements (2005) estimates that the daily turnover in the global foreign exchange market was valued at 840 billion U.S. dollars in 1992, 1590 billion U.S. dollars in 1998, and 1880 billion U.S. dollars in 2004.
} 
indicative data available from, for example, central banks, since they are simply more representative of the true exchange rates that prevailed in the market.

We generate forecasts for the last $N=3000$ observations of GBPUSD, USDCHF, USDJPY, and USDCAD. For EURUSD, EURCHF, EURJPY, and EURGBP we generate forecasts for the last $N=1000$ observations, since these pairs only have 2019 observations available. Thus, the time index of the first forecast is $T_{0}=1086$ for the former group of currency pairs, and $T_{0}=1020$ for the latter group. We reserve the initial observations to ensure that there is enough data for our estimation sample selection procedures that are described in a later section.

All of the forecasts in this paper are real-time, out-of-sample, one-day-ahead forecasts. The forecasts are real-time since we only use information that was available at the time that the forecast would have been historically generated. We place significant emphasis on this exclusive use of ex-ante information. Use of any ex-post information in producing forecasts or use of intermediate results that are based on ex-post information ${ }^{4}$ can lead to severe forecast bias.

It is common in the literature to forecast the natural log first difference of the exchange rate, which corresponds to the log one-period return ${ }^{5}$ of the exchange rate. We will demonstrate later in this paper that this common transformation can result in forecasts that are worse than those based on the non-transformed series. We let $s_{t}$ denote

\footnotetext{
${ }^{4}$ An example would be to estimate hyper-parameters (such as the optimal linear combination of two forecasts) over the full sample, and then use this information to improve the (previously) out-of-sample forecasts.

${ }^{5}$ Note that this return corresponds to holding the currency pair long (i.e.: buying the base currency using the quoted currency).
} 
the nominal exchange rate and let $\Delta s_{t}=s_{t}-s_{t-1}$ denote the first difference of the nominal exchange rate. Similarly, we let $l_{t}$ denote the natural logarithm of the nominal exchange rate, and denote the first difference of this series as $\Delta l s_{t}=l s_{t}-l s_{t-1}$. Hence, we are interested in producing one-day-ahead forecasts for both $\Delta s_{t}$ and $\Delta l s_{t}$. Summary statistics for these two variables for each of the eight currency pairs over the full samples are presented in Tables 1.1 and 1.2. Of important note is that the kurtosis is positive for all of the currency pairs, which implies that the distribution tails are heavier than those of the normal distribution. This confirms the findings of Boothe \& Glassman (1987b) and Hsieh (1988) that exchange rate series display leptokurtosis, and is our motivation for the use of the LAD estimator described in the next section.

We produce a forecast, $f_{T+1}$, of either $\Delta s$ or $\Delta l s$ at time $T+1$ using information up to time $T$. More precisely, we use a sample of $n_{T}$ observations prior to and including the observation at time $T$ to produce this forecast. We denote the sample size as $n_{T}$ since we allow the value of $n$ to be conditional on $T$. The selection of $n_{T}$ for each value of $T$ is described in section 5 . Thus, to produce a forecast for $\mathrm{T}+1$ we use observations from $T$ $n_{T}+1$ to $T$. Similarly, to produce a forecast for $T+2$, we use observations from $(T+1)-n_{T}+1$ to $(T+1)$. We continue in this way until we have generated $N$ forecasts. 


\section{Models and Estimators}

As the focus of this paper is on robust forecasting methodology, we limit the complexity of the models to a simple linear first-order autoregressive specification. Specifically, we generate forecasts using the following model:

$$
\Delta s_{t}=\alpha_{0}+\alpha_{1} \Delta s_{t-1}+\varepsilon_{t},
$$

where $\Delta s_{t}$ is the first difference of the nominal exchange rate and $\varepsilon_{t}$ is an error term. We will often refer to this model as the " $\Delta s_{t}$ model" in the remainder of this paper. Since we are also interested in determining the value of applying a logarithmic transformation to the data, we also produce forecasts for the following specification:

$$
\Delta l s_{t}=\beta_{0}+\beta_{1} \Delta l s_{t-1}+e_{t},
$$

where $\Delta l_{t}$ the first difference of the natural $\log$ nominal exchange rate and $e_{t}$ is an error term. We refer to this model as the " $\Delta l s_{t}$ model." In addition to these two autoregressive specifications, we will also consider the corresponding random walks with drift as benchmark models. These models are obtained by setting $\alpha_{1}$ equal to zero in (1) and $\beta_{1}$ equal to zero in (2).

We consider three estimators of the models given in (1) and (2). The first estimator that we consider is the usual OLS estimator. As is generally the case in the literature, this estimator has been widely applied in forecasting contexts. OLS is not considered to be robust to outliers due to the large influence that these outliers have on the sum of squared errors.

The second estimator that we consider is maximum likelihood estimation with tdistributed errors (referred to as MLE-t throughout the paper). Lange, Little \& Taylor 
(1989) examine the use of t-distributed errors to produce estimates that are robust to outliers. We allow the degrees of freedom, also known as the kurtosis parameter in the context of robust estimation, to vary continuously.

The third estimator that we consider is least absolute deviations (LAD), also known as Laplace estimation or L1 estimation (see Judge et al. (1988) for an overview). LAD can also be thought of as a special case of quantile regression, where the quantile that we estimate is the median. The LAD estimates of, for example, $\alpha_{0}$ and $\alpha_{1}$ in model (1) over a sample of $n$ observations are a solution to the following minimization problem:

$$
\min _{\alpha_{0}, \alpha_{1}} \sum_{t=1}^{n}\left|\Delta s_{t}-\alpha_{0}-\alpha_{1} \Delta s_{t-1}\right|
$$

It is important to note that there may be multiple solutions to this minimization problem, and consequently LAD estimates are not always unique as with OLS. LAD is typically a superior estimator to OLS when the distribution of the errors of a model displays leptokurtosis. LAD estimates will be less sensitive to outliers because the absolute loss of an outlier will be less than the squared loss under OLS. Consequently, LAD estimates of a model are considered to be robust to outliers. We compute LAD estimates using iteratively reweighted least squares.

Each of the three estimators that we consider can be translated to an equivalent maximum likelihood procedure. In the case of OLS, the estimates correspond to maximum likelihood estimation of the model parameters with a normally distributed error term. Similarly, for MLE-t, the corresponding maximum likelihood procedure is for a model with a t-distributed error term. Finally, the LAD estimates are equivalent to 
parameters that maximize a Laplace ${ }^{6}$ likelihood function. The exact form of each of the likelihood functions can be found in Appendix C.

\section{LAD Estimation with Bounded Influence}

In this section we explore the possibility of improving model estimates, and consequently forecasts, by using bounded influence techniques. Bounded influence is a simple and practical method for removing noise from a time series in order to produce superior estimates of model parameters. In particular, we apply methodology developed by Krasker \& Welsch (1982) to develop a two-stage LAD estimation procedure.

Krasker \& Welsch (1982) develop a bounded influence regression estimator that is asymptotically efficient. Effectively, their procedure is a weighted least squares estimator, where the weights are calculated so as to bound the influence of outliers in both the dependent variable and the regressors. While not originally developed for use with autoregressive time series models, Krasker \& Welsch (1983) note that their bounded influence procedures are resistant to both additive and innovation outliers in time series models (see Martin (1979) for an explanation of these terms). We choose to apply the Krasker-Welsch method since it is both simple to implement and completely data driven.

As Krasker \& Welsch (1982) note, the diagnostic information provided by their bounded influence procedure can be used to locate potential outliers. In the first stage of

\footnotetext{
${ }^{6}$ The Laplace distribution is also commonly known as the double exponential distribution.
} 
our procedure, we use the Krasker-Welsch diagnostic information to construct a bounded influence estimation sample. The second stage consists of simply applying the LAD estimator to this new estimation sample. It is important to note that what we propose here is not a true bounded influence LAD estimator. This would be a more complicated alternative that would require the development of an appropriate weighted LAD procedure that, to the best of the author's knowledge, does not exist at present.

First, we define the diagnostic statistic DFFITS (see Belsley, Kuh, \& Welsch (1980) for more information on this statistic) as follows:

$$
\operatorname{DFFITS~}_{t}=\frac{h_{t} e_{t}}{s(t)\left(1-h_{t}\right) \sqrt{h_{t}}},
$$

where $h_{t}$ is the $t$-th diagonal element of the $n_{T}$ by $n_{T}$ hat matrix $H=X\left(X^{\prime} X\right)^{-1} \mathrm{X}^{\prime}, X$ is the $n_{T}$ by 2 matrix of regressors, $e_{t}$ is the $t$-th estimated OLS residual, and $s(t)$ is the sample standard deviation of $e_{t}$ with the $t$-th observation removed.

Krasker, Kuh \& Welsch (1983) show that to bound the influence of observations in our sample for a given bound on the sensitivity, $c$, we require that

$$
\left(n_{T}-1\right)^{1 / 2} \mid \text { DFFITS } \mid \leq c .
$$

Hence, we use this inequality to determine which observations to keep in the sample. We call this reduced sample the bounded influence (BI) sample. We apply the LAD estimator to the BI sample, and denote the resulting estimates as LAD-BI estimates.

A significant point of contention is the selection of the value of the bound on the sensitivity, $c$. This value is more generally known as a "tuning constant" in the literature. A number of methods for choosing $c$ are proposed by Peters, Samarov \& Welsch (1981), 
Krasker \& Welsch (1982), Kelly (1992), Kelly (1996), Warwick (2005), and Warwick \& Jones (2005). Krasker, Kuh \& Welsch (1983) point out that a bound value of around two is a good choice for the Krasker-Welsch estimator for diagnostic purposes. However as Kelly (1992) shows, the choice of the tuning constant is extremely important in the tradeoff between estimator variance and estimator bias. Kelly (1992) also demonstrates that the optimal tuning constant can be significantly different from two. Kelly (1996) presents an adaptive method based on minimizing the jack-knife-estimated asymptotic mean squared error. Along similar lines, Warwick (2005) and Warwick \& Jones (2005) describe a data-driven process that the selects the tuning constant by minimizing the asymptotic mean squared error.

Our requirements for the tuning constant are very well defined since we know that we wish to reduce the sample in such a way as to produce estimates that will in turn produce better forecasts as measured by the corresponding returns. It therefore makes sense that we should attempt to estimate the value of the bound based on this information. Consequently, we expand on ideas from Kelly (1996), Warwick (2005), and Warwick \& Jones (2005) and propose a bootstrap-based method that selects the tuning constant by maximizing out-of-sample returns ${ }^{7}$. Our procedure uses the $T_{0}-1$ initial observations for which we do not produce forecasts (ex-ante data) to estimate the tuning constant ${ }^{8}$. In

\footnotetext{
${ }^{7}$ Note that we do not simply find the value of the bound that minimizes the in-sample returns, since we believe there is a serious risk of over-optimization in a forecasting context. The risk is that we could select a value of the tuning parameter that is optimal for the current sample, but performs badly out of sample.

${ }^{8}$ Instead of estimating the tuning constant only once using the initial sample of $T_{0}-1$ observations, we could recursively estimate the tuning constant by repeating the bootstrap procedure for each value of $T$. However, since a relatively large number of bootstrap
} 
particular, each bootstrap replication draws 20 block samples of random size, and computes the one-step-ahead out-of-sample LAD-BI forecasts for each of these block samples for a range of values of $c$. We then find the mean return over the 20 block samples and select the value of $c$ that maximizes this quantity for each replication. The mean of the values of $c$ that we obtain over the bootstrap replications is our estimate of the tuning constant. The exact details of the bootstrap procedure that we use can be found in Appendix D.

As an alternative to the bound being a fixed constant, it is also possible that the bound is a function of descriptive statistics of the estimation sample. In particular, it is intuitive that the bound be a function of a measure of the heaviness of the sample's distribution tails, since such a measure should be indicative of the level of outlier contamination in the sample. Thus, we estimate the following linear model of the tuning constant by OLS over the $I$ bootstrap replications:

$$
c_{i}=\varphi_{0}+\varphi_{1} k_{i}+u_{i},
$$

where $c_{i}$ is the tuning constant the maximizes the mean return for bootstrap replication $i$, and $k_{i}$ is the mean sample kurtosis over the 20 block samples for bootstrap replication $i$.

replications (at least 1000) are needed, such a procedure would be extremely computationally intensive when there are 3000 different values of $T$. 


\section{Estimation Sample Selection}

One forecasting problem that we face is that we are not sure how much previous information we should use in estimating the model of interest. Specifically, since we are using univariate autoregressive models, we need to choose the number previous observations of the series to include in the estimation sample. It is important to note that this sample size can depend on $T$, so we denote it by $n_{T}$, as mentioned earlier.

A common solution to the estimation sample size selection problem is to simply use as much data as is available at time $T$, so that $n_{T}=T$. This approach is commonly known as the "expanding window" method, since the sample size expands by one observation for each subsequent one-step-ahead forecast.

There are some cases where the expanding window method will not be optimal. In particular, model instability may mean that parameter estimates based on all available data are biased relative to their true values at time $T$. Pesaran \& Timmermann (2002) investigate this problem in the context of structural breaks in the time series of interest. They suggest using a reverse ordered Cusum, based on methodology developed by Brown, Durbin \& Evans (1975), to locate the most recent break point and then use the post-break data to estimate the model and generate a forecast. Pesaran \& Timmermann (2005) extends the authors' earlier work to the realm of autoregressive models. They find that including pre-break data can reduce the bias of the parameter estimates in some cases and suggest using the expanding window method in most cases.

Another alternative is to use a rolling window, where the estimation sample remains the same size for each forecast horizon. This implies that only recent data will be used in 
the estimation of the model, i.e. $n_{T}=n_{0}$ where $n_{0} \leq T_{0}$ is a constant. However, this does not completely alleviate the problem of biased parameter estimates in the presence of a structural break. As Pesaran \& Timmermann (2002) note, if the structural break occurs at time $T-b$ and we take a rolling window of size $n_{0}$, where $n_{0}>b$, then we will have $n_{0}-b-1$ observations from before the break point included in the estimation sample. We could ensure that we do not have many observations prior to a possible break point by taking a very small value of $n_{0}$, but this would introduce significant small sample bias to the parameter estimates.

It is important to note that there is a difference between a structural break that can be detected as a statistically significant change in model parameters and model instability with respect to forecasting a time series. It is often the case that very small statistically insignificant breaks in the parameter values can have a measurable impact on the value of the forecast. Pesaran \& Timmermann (2007) suggest using a pseudo-out-of-sample cross-validation method or combining forecasts ${ }^{9}$ across different rolling window sizes when breaks are very small.

Expanding on Pesaran \& Timmermann (2005 \& 2007), we propose a new nonparametric sample size selection approach ${ }^{10}$. The approach is nonparametric since it does not require the specification of a forecasting model or any distributional

\footnotetext{
${ }^{9}$ We explore the value of forecast combinations in section 10 .

${ }^{10}$ We applied the pseudo-out-of-sample method that Pesaran \& Timmermann (2007) propose, but found that our in-sample nonparametric method was substantially better over the series that we consider. A formal comparison of the two methods would be quite involved, and is beyond the scope of this paper.
} 
assumptions. This is advantageous since it implies that the samples selected using this method can be used with any model of interest.

The intuition of our method is based on the possibility that there exists a statistically insignificant structural break at some time prior to time $T$. We wish to determine the optimal number of previous observations of the series $\left(n_{T}\right)$ that we should use to estimate the model, and consequently, to generate the forecast of the series at time $T+1$. Since Pesaran \& Timmermann (2005) indicate that using pre-break data can improve parameter estimates when using autoregressive models, we do not attempt to precisely estimate the nearest break point. In fact, we leave open the possibility that there may be two or more break points prior to time $T$, and allow for estimation samples that could include data before these break points, if it is optimal to do so.

We find the optimal estimation sample size by locating the absolute minimum ${ }^{11}$ of a pre-specified in-sample loss function, $L\left(n_{T}\right)$, to determine the optimal number of estimation sample observations. For example, consider a sample of $n_{l}$ observations. If $L\left(n_{2}\right)<L\left(n_{1}\right)$, then there is an average gain to using $n_{2}$ observations relative to $n_{1}$ observations. Conversely, if $L\left(n_{2}\right)>L\left(n_{1}\right)$, then there is an average loss to using $n_{2}$ observations, relative to $n_{1}$ observations.

The specific loss functions that we consider are the mean absolute error (MAE) and mean squared error (MSE). For example, using the first differenced nominal exchange rate $\left(\Delta s_{t}\right)$, the loss functions are:

\footnotetext{
${ }^{11}$ It is also possible to proceed in a marginal loss fashion by searching for the local minimum closest to $T$. This method is more complex since it requires formal identification local minima over discrete data, with some tolerance for "false" minima. As such, we leave this for future research.
} 


$$
\begin{gathered}
L_{1}\left(n_{T}\right)=\frac{1}{n_{T}} \sum_{t=T-n_{T}}^{T}\left|\Delta s_{t}-\hat{\mu}_{0}\right|, \\
L_{2}\left(n_{T}\right)=\frac{1}{n_{T}} \sum_{t=T-n_{T}}^{T}\left(\Delta s_{t}-\hat{\mu}_{1}\right)^{2},
\end{gathered}
$$

where $\hat{\mu}_{0}$ is the median of $\Delta s_{t}$ over the $n$ observations, and $\hat{\mu}_{1}$ is the mean of $\Delta s_{t}$ over the $n$ observations. We choose $n_{T}$ by minimizing $L_{1}\left(n_{T}\right)$ or $L_{2}\left(n_{T}\right)$ over all $n_{T}=50, \ldots, T$. We do not consider values of $n_{T}$ less than 50 in order to ensure that samples with extreme small sample bias are not included. Additionally, the values of the loss functions typically vary erratically for values of $n_{T}$ less than 50 , which can result in improper selection of the absolute minimum, as noted by Pesaran \& Timmermann (2007).

There is also an equivalent of this nonparametric method in the context of parametric maximum likelihood estimation. It can be shown that minimization of $L_{2}\left(n_{T}\right)$ is equivalent to maximizing the mean likelihood of a random walk model with drift and normally distributed errors. Similarly, minimization of $L_{1}\left(n_{T}\right)$ is equivalent to maximizing the mean likelihood of a random walk model with drift and Laplace distributed errors. Hence, the parameter estimates obtained using the selected samples can be thought of as maximum likelihood estimates over both the parameter space and the sample space. Based on this, we expect that the samples selected using the MAE loss function, $L_{1}\left(n_{T}\right)$, will produce the best LAD parameter estimates, and that the samples selected using the MSE loss function, $L_{2}\left(n_{T}\right)$, will produce the best OLS parameter estimates.

It is possible to replace the random walk model in the parametric version with the autoregressive models described in (1) and (2). In fact, we could replace the random 
walk model with any specific forecasting model of our choice, in order to select the optimal samples for this particular model. The key advantage of the nonparametric version of the sample selection procedure is that it does not depend on a pre-specified model, by definition. As such, the samples selected by this procedure can be used with any forecasting model, and samples do not need to be reselected when we change models. This particular feature can be useful for comparing performance across models using the same estimation samples. Furthermore, if the best model is unknown, the nonparametric procedure can be used to find a first-stage solution to the joint sample-size uncertainty and model uncertainty problem. Consequently, we will demonstrate and use the nonparametric version of the sample selection procedure in this paper, even though we do not specifically consider model selection here.

\section{Evaluation of Out-of-Sample Forecasts}

In the previous sections we explored some of the potential problems with the usual estimation sample selection methods and the usual model estimators. In the next section we will explore the consequences of using these estimators and sample selection methods. Prior to doing this, we define several descriptive statistics that we will use evaluate the forecasts.

The usual statistic in the literature for evaluating the forecasting ability of a given model is the mean squared error (MSE), defined as: 


$$
M S E=\frac{1}{N} \sum_{t=T_{0}}^{T_{0}+N-1}\left(\Delta s_{t}-f_{t}\right)^{2}
$$

In this paper, we take the square root of this quantity and refer to this as the root mean squared error (RMSE). The primary reason for taking the square root is so that the units of measurement are the same as those for the mean absolute error (MAE), which we define as:

$$
M A E=\frac{1}{N} \sum_{t=T_{0}}^{T_{0}+N-1}\left|\Delta s_{t}-f_{t}\right|
$$

Note that both the RMSE and MAE definitions are adjusted accordingly when we evaluate forecasts based on the log first differenced exchange rate. Effectively, this means that we replace $\Delta s_{t}$ in equations (9) and (10) with $\Delta l s_{t}$.

The RMSE and MAE statistics do not necessarily measure the value of the forecasts from a market efficiency perspective. If the foreign exchange market is inefficient we would expect to be able to generate an excess return by trading currency pairs.

Consequently, the return on foreign exchange transactions signaled by our forecasts is very relevant. We define the percentage correct $(\mathrm{PC})$ statistic as the percentage of forecasts that correctly predict the sign of the change in the exchange rate:

$$
P C=100\left[\frac{1}{N} \sum_{t=T_{0}}^{T_{0}+N-1} I\left(\Delta s_{t}^{*} f_{t}\right)\right], \quad I(x)=\left\{\begin{array}{ll}
1 & \text { if } x \geq 0 \\
0 & \text { if } x<0
\end{array}\right. \text {. }
$$

Next, the gross annualized return (GAR) is the return on trading the currency based on the sign of the forecast. In particular, if the forecast for time $T+1$ is positive, we take a long position in the currency pair (i.e. we buy the base currency using the quoted currency). Similarly, if the forecast for $T+1$ is negative, we take a short position in the 
pair (i.e. we buy the quoted currency using the base currency) ${ }^{12}$. This information can be summarized in the following function:

$$
\operatorname{signal}_{t}\left(f_{t}\right)=\left\{\begin{array}{ll}
+1 & \text { if } f_{t} \geq 0 \\
-1 & \text { if } f_{t}<0
\end{array}\right. \text {. }
$$

The daily log return on the currency pair is then:

$$
\text { ret }_{t}=\operatorname{signal}_{t}\left(f_{t}\right)\left(\ln \left(s_{t}\right)-\ln \left(s_{t-1}\right)+\left[\ln \left(1+i_{t-1}^{B}\right)-\ln \left(1+i_{t-1}^{Q}\right)\right]\right),
$$

where $i_{t}$ is the interest rate at time $t$, and B and Q denote the base currency and quoted currency, respectively ${ }^{13}$. Hence, the GAR is defined as:

$$
G A R=\left[\prod_{t=T_{0}}^{T_{0}+N-1}\left(1+r e t_{t}\right)\right]^{D / N}-1
$$

where $D$ is the average number of trading days per year $(D=258$ for our data set).

It is important to include interest rates in the calculation of the daily log return, and consequently, the calculation of the GAR. The uncovered interest parity theory suggests that movements in nominal exchange rates should be matched by equivalent adjustments in interest rates. If this theory holds, correct forecasts of the nominal exchange rate would be offset by the interest rate differential, and we would expect the average return to be equal to zero. As a result, a positive (and statistically significant) GAR implies that uncovered interest parity does not hold, since the return is in excess of the interest rate differential. Since we are interested in searching for market inefficiencies, the GAR will be our primary statistic for evaluation of forecasts throughout this paper.

\footnotetext{
${ }^{12}$ It is important to note that we do not allow the trader to take a "no position" stance, which could significantly increase the GAR if we were able to avoid particularly bad trades.

${ }^{13}$ Detailed information on the interest rates that we use can be found in Appendix B.
} 


\section{Forecasting Results}

In Table 2, we present the results for the benchmark random walk models with drift. We estimate these models by OLS over an expanding window, as this is most common in the literature. This table contains results based on forecasting the first differenced nominal exchange rate $\left(\Delta s_{t}\right)$ and results based on forecasting the log first differenced series $\left(\Delta l s_{t}\right)$. We note that the PC statistics are close to $50 \%$ for all currency pairs and portfolios, and that the GARs are negative in all cases. In all subsequent tables, we present RMSE and MAE statistics as a ratio of those obtained by the benchmark random walk model. As such, an RMSE ratio (MAE ratio) less than one implies the RMSE (MAE) is less than that of the benchmark model. We do not present the PC or GAR statistics relative to the random walk since more practical and obvious benchmarks are available. In particular, for the PC statistic, the obvious benchmark is $50 \%$, which would imply the model's predictions are correct as often as they are incorrect. For the GAR statistics, the obvious benchmark is a GAR of zero, since greater than this value implies positive profit and less than this value implies negative profit.

Table 3 presents results for forecasts based on rolling estimation windows of sizes $n_{T}=100,500$, and 1000 for the eight currency pairs and the three basic estimators. For this table, sub-tables beginning with 3.1 present results based on the $\Delta s_{t}$ model, and subtables beginning with 3.2 present results based on the $\Delta l s_{t}$ model. Tables 4 and 5 also follow this numbering scheme. For all six sub-tables of Table 3 we see that the RMSE and MAE statistics are very similar across estimators and sample sizes. Both of these statistics are generally close to one in all cases. We note that the portfolio PC and GAR 
statistics for the LAD-based forecasts are always larger than those of either the OLS forecasts or the MLE-t forecasts, with similar performance for the $\Delta s_{t}$ model and the $\Delta l s_{t}$ model.

Table 4 contains results for forecasts based on an expanding estimation window. Again, we see that the RMSE and MAE statistics are very similar across estimators. For both the $\Delta s_{t}$ model and the $\Delta l s_{t}$ model, we see that the OLS-based forecasts produce a negative portfolio GAR. The GARs for the individual currency pairs also vary substantially for the OLS-based forecasts. We see that the LAD-based forecasts once again produce the largest portfolio GARs. Looking at individual currency pairs, the LAD-based forecasts produce a positive GAR for seven of the eight pairs for both models.

We draw several inferences from both the rolling window and expanding window results. First, it is clear that low RMSE or low MAE does not necessarily indicate a large GAR. Often both the RMSE and MAE ratios are greater than one, while the GAR statistics are significantly better than the benchmark model. Furthermore, between estimators, using a rolling estimation window of 500 observations and the $\Delta s_{t}$ model for EURCHF, we see that the GAR of the LAD-based forecasts is $1294 \%$ larger than that of the OLS-based forecasts, but the RMSE and MAE ratios are larger for the LAD-based forecasts relative to their OLS-based counterparts. These results are supportive of Boothe \& Glassman (1987a), who show that models that perform well in terms of accuracy measures will not necessarily produce the largest excess returns. 
Second, though the PC statistic is more closely correlated with the GAR, we should be aware that a larger value of PC may not imply a larger GAR. For example, using a rolling estimation window of 500 observations and the $\Delta s_{t}$ model for GBPUSD, we see that the GAR of the LAD-based forecasts is larger than that of the OLS-based forecasts, but that the reverse is true for the PC statistics. The intuition behind this is that the model may predict many small changes in the exchange rate correctly, but fail to predict larger changes, resulting in a large value of PC and a small GAR.

Third, we note that the portfolio GAR for the OLS-based forecasts is often very small or negative. Though MLE-t-based forecasts do improve on this, even these are dominated by the LAD-based forecasts in all cases.

Fourth, we see that the expanding window and rolling windows of particular sizes perform similarly. In this case, the expanding window would have an advantage, since the optimal rolling window size is unknown ex-ante. Finally, there does not appear to be a clear advantage to using either the $\Delta s_{t}$ model or the $\Delta l s_{t}$ model, as results for both are very similar.

Next, we turn our attention to the results contained in Table 5. This table presents results for the optimal estimation samples selected using the nonparametric procedure that was described in section 5 . In all four sub-tables of Table 5 we see that the LAD estimator continues to provide superior forecasts relative to those produced by the OLS and MLE-t estimators. Consequently, our primary focus will be the LAD-based forecasts. For the $\Delta s_{t}$ model, we see a substantial GAR improvement of nearly $33 \%$ for the LAD-based forecasts over those for the expanding window method when we optimize 
the estimation sample using MAE. Similarly, these forecasts outperform the best rolling window by about $17 \%$ in terms of GAR. It is also clear that optimization based on MAE is preferable to optimization based on MSE for the $\Delta s_{t}$ model, since the former produces larger GARs for each of the three estimators. For the $\Delta l s_{t}$ model, we see that the forecasts based on the MSE-optimized samples perform about equally as well as those from on the MAE-based samples. We note that the RMSE and MAE ratios in Table 5 are almost always larger than one, even though the GAR statistics are substantially larger than those obtained using the benchmark model. This further supports our previous conclusion that accuracy measures are not highly correlated with returns.

In terms of LAD-based forecast portfolio GARs, the $\Delta s_{t}$ model appears to perform the best across the results in the four sub-tables of Table 5. In particular, the portfolio GAR for LAD-based forecasts with the estimation sample optimization based on MAE are over $20 \%$ larger for the $\Delta s_{t}$ model, relative to the $\Delta l s_{t}$ model. As such, we will use the $\Delta s_{t}$ model exclusively throughout the remainder of this paper.

Thus far, we have shown that the LAD estimator produces forecasts that consistently outperform those based on both the OLS and MLE-t estimators when measured by GAR. Additionally, we have demonstrated that our non-parametric datadriven sample selection procedure selects estimation samples that produce forecasts with a larger GAR than that of forecasts produced using a rolling or expanding window.

Table 6 presents the forecasting results of the LAD-BI estimator developed in section 4. Results based on a fixed tuning constant are denoted by FTC, whereas results based on tuning constant adjusted for estimation sample kurtosis, using the estimated 
equation (6), are denoted by KTC. We note immediately that the portfolio GARs when using optimal samples based on MAE are substantially larger than their counterparts for optimal samples based on MSE. Thus, we focus on the results from using the MAEoptimized samples. For the FTC results, we see an overall improvement in the portfolio GAR by over $12 \%$. For the individual currency pairs, five GARs are improved over the original LAD-based forecasts, while three GARs are worse. For the KTC results, there is an overall improvement in the portfolio GAR by about $25 \%$ relative to the portfolio GAR of the original LAD-based forecasts. Seven of the individual currency pairs show an improved GAR, with an average individual improvement of $48 \%$ over these seven pairs.

Of note, for both the FTC and KTC results, we see that USDCAD goes from having a positive GAR of $2.55 \%$ to a negative GAR. This likely occurs because the tuning constant, and the relationship in equation (6), are estimated only over the first $T_{0}-1$ observations. It is very possible that there is a structural change in USDCAD over the sample period that causes the estimated tuning constant to be biased from the optimal value after a certain point in the series. Such a problem could be resolved by recursively estimating the tuning constant and its relationship with the estimation sample kurtosis. As this would be extremely computationally intensive, we leave this for future research.

\section{Statistical Significance}

In the previous section we were able to show that some of our models and estimators predict the sign of the forecast change quite well and can produce reasonably large gross 
returns. In this section we attempt to determine whether the results that we obtain are statistically significant. We consider three different approaches to the issue of statistical significance. The first is the predictive accuracy test of Pesaran \& Timmermann (1992), which determines whether the forecasts have any market timing ability relative to the exchange rate series that they attempt to predict. The second approach is to utilize the Diebold-Mariano (1995) statistic, and subsequent developments based on this statistic, to determine if the returns generated by our methods are statistically better than those produced by using a naïve forecasting model. The final method is to determine the probability of obtaining a GAR as large as we do when the exchange rate is a random walk process.

The Pesaran \& Timmermann (1992) nonparametric test of predictive accuracy tests the null hypothesis that the forecasts $\left(f_{t}\right)$ and the series that they attempt to predict $\left(\Delta s_{t}\right)$ are independently distributed. Rejection of this null hypothesis implies that the forecasts have some predictive power. Pesaran \& Timmermann (1994) show that this test is asymptotically equivalent to the Henriksson \& Merton (1981) test of market timing. In order to define the Pesaran-Timmerman test statistic, we first define the following:

$$
p_{x}=\frac{1}{N} \sum_{t=T_{0}}^{T_{0}+N-1} I\left(f_{t}\right), \quad p_{y}=\frac{1}{N} \sum_{t=T_{0}}^{T_{0}+N-1} I\left(\Delta s_{t}\right), \quad p=\frac{1}{N} \sum_{t=T_{0}}^{T_{0}+N-1} I\left(\Delta s_{t}^{*} f\right),
$$

where $I(x)$ is the indicator function as defined in section 6 . These statistics correspond to the empirical probability that the forecast is positive, the empirical probability that the change in the exchange rate is positive, and the empirical probability that their product is positive, respectively. If we let $p_{*}=p_{y} p_{x}+\left(1-p_{y}\right)\left(1-p_{x}\right)$ then the PesaranTimmermann test statistic is given by: 


$$
P T=\frac{p-p_{*}}{\left(\operatorname{var}(p)-\operatorname{var}\left(p_{*}\right)\right)^{1 / 2}},
$$

where $\operatorname{var}(p)=N^{-1} p_{*}\left(1-p^{*}\right)$ and $\operatorname{var}\left(p_{*}\right)=N^{-1}\left(2 p_{y}-1\right)^{2} p_{x}\left(1-p_{x}\right)+N^{-1}\left(2 p_{x}-1\right)^{2} p_{y}\left(1-p_{y}\right)$ $+4 N^{-2} p_{y} p_{x}\left(1-p_{y}\right)\left(1-p_{x}\right)$. The $P T$ statistic asymptotically follows a standard normal distribution. Asymptotic convergence does not depend on the specific underlying probability distribution, and only requires that the probability of changes in the direction of $f_{\mathrm{t}}$ and $\Delta s_{t}$ is time invariant and does not take the extreme values of 0 or 1.

The Diebold-Mariano (1995) test is used to compare the forecast errors of two competing models with respect to a given loss function. Much of the recent literature on comparing forecast accuracy is based on this statistic. Specifically, if we define the difference in loss from the forecasts as $d_{t}=L\left(\Delta s_{t}, f_{1, t}\right)-L\left(\Delta s_{t}, f_{2, t}\right)$, where $f_{1, t}$ is the forecast from the OLS-estimated random walk benchmark model, $f_{2, t}$ is the forecast from the LAD-BI-estimated $\Delta s_{t}$ model, and $L$ is a given loss function, then we can test the null hypothesis that $E\left(d_{t}\right)=0$. This null hypothesis corresponds to the two models having equal forecast accuracy.

Harvey, Leybourne, and Newbold (1997) show that the Diebold-Mariano test tends to be oversized in small to moderate samples. They show that this is particularly the case if the forecast errors come from a fat-tailed distribution, which is quite likely the case for our series. In a later work, Harvey, Leybourne, and Newbold (1999) propose a modified Diebold-Mariano test statistic that protects against incorrect rejection of the null hypothesis in the presence of fat-tailed forecast error distributions. As a result, we use 
this test statistic to evaluate the out-of-sample performance of our models. This DieboldMariano test statistic is given by:

$$
D M=\left[N+1-2 q+\frac{q(q-1)}{N}\right]^{1 / 2}\left[\frac{\frac{1}{n} \sum_{t=T_{0}}^{T_{0}+N-1} d_{t}}{\left(\gamma_{0}+2 \gamma_{1}+\ldots+2 \gamma_{q-1}\right)^{1 / 2}}\right],
$$

where $q=\operatorname{int}\left(0.5 N^{1 / 3}\right)+1, d_{t}=L\left(\Delta s_{t}, f_{1, t}\right)-L\left(\Delta s_{t}, f_{2, t}\right)$, and $\gamma_{i}$ is the $\mathrm{i}$-th autocovariance of the $d_{t}$ series. We use a returns-based loss function, $L\left(\Delta s_{t}, f_{t}\right)=-\operatorname{ret}_{t}\left(\Delta s_{t}, f_{t}\right)$, since we are interested in determining if the gross returns from the LAD-BI-estimated $\Delta s_{t}$ model are statistically larger than the gross returns of the OLS-estimated random walk benchmark model.

The $D M$ statistic is asymptotically normally distributed under certain assumptions, but these assumptions are rarely satisfied in finite samples with estimated models. Clark and McCracken (2001) and McCracken (2004a) show that in the case of nested models, Diebold-Mariano-type tests have non-standard limiting distributions under the null hypothesis of equal forecast accuracy. McCracken (2004a) provides the asymptotic distribution of the $D M$ statistic (called OOS-t in his paper) for one-step-ahead forecasts. This asymptotic distribution depends on the number of additional parameters in the alternative model relative to the null (random walk) model, and the ratio of the number of forecasts $(N)$ to the number of observations used to estimate the model for the first forecast.

Clark and McCracken (2005) present Monte Carlo evidence on size and power for various forecast horizons for both the simulated asymptotic distribution and for a simple 
bootstrap of a DM-type statistic. Their results indicate that both the simulated asymptotic test and the bootstrapped test have slight size distortions ${ }^{14}$. Inoue \& Kilian (2004) point out that bootstrap versions of tests of forecast accuracy have a proven performance record in alleviating potential size problems. As a result, we use a bootstrap procedure to generate p-values for the DM test statistics. The full bootstrapping algorithm that we use is described in Appendix E.

The final test that we consider is a simple returns-based test. In particular, we determine the probability of having GARs as large as those we obtain if the exchange rate follows a random walk process. We obtain this probability by forming the empirical distribution of GARs under the null hypothesis of a random walk generated exchange rate. This null hypothesis is the same as for the DM test described above. As such, in the bootstrap procedure for generating the distribution of the DM statistic, we save the GAR of the estimated $\Delta s_{t}$ model for each bootstrap replication to obtain the empirical distribution of GARs.

Table 7 presents Pesaran-Timmermann statistics, Diebold-Mariano statistics, GARs, and corresponding p-values for the LAD and LAD-BI forecasts based on MAEoptimized estimation samples. The results for the LAD-based forecasts in sub-table 7.1 have PT statistics that are significant at the $5 \%$ level for the four currencies where we have a large sample of 3000 forecasts. Similarly, the DM statistics are significant at the

\footnotetext{
${ }^{14}$ McCracken (2000) and McCracken (2004b) also point out that the Diebold-Mariano statistic can be improperly sized in some situations where we use parameter estimates instead of the true parameter values. Based on a small Monte Carlo simulation (results not presented here for brevity), we found that the bootstrapped DM test has size properties very similar to those reported by Clark \& McCracken (2005).
} 
$5 \%$ level for the first five listed currencies, and the GAR statistics are highly significant for the first three listed currencies.

The statistical significance of the results generally improves (the exception being USDCAD) as we move to sub-table 7.2 which contains results for forecasts based on the LAD-BI (KTC) estimator. For this estimator, five of the six currency pairs where we obtain a positive GAR have PT, DM, and GAR statistics that are significant at the $5 \%$ level. The notable exception is EURJPY, which has a large positive return, but none of the statistics are significant at the 5\% level. However, the DM statistic for EURJPY is significant at the $10 \%$ level.

Overall, we find that the DM and GAR statistics indicate that there is substantial evidence that the returns obtained by the LAD-BI estimator due to market inefficiencies are highly statistically significant for the majority of currency pairs. In addition, the significant PT statistics indicate that the forecasts display evidence of market timing ability.

\section{Profitability and Risk}

Several authors (Meade (2002) and Sarantis (2006), for example) point out that even though an exchange rate forecasting model can generate statistically significant profits in theory, there are several practical factors that must be accounted for to demonstrate real historical profitability. The two most common issues that arise are that we assume that 
we can trade at the quoted price, and that we would encounter significant transactions costs when trading.

We believe that the first of these two issues is not very significant for two reasons. First, as we mentioned earlier, our data are historical quoted rates. As such, a trader who was receiving these exchange rates in real-time could have placed trades at these rates. However, this does not completely alleviate the potential timing issue. The problem is that the trader requires the present day close price before he can input this information into his forecasting model and determine what trade to make over the next day. Hence, there may be some variability between the current day close price and the price at which we enter into the next trade (assuming our forecast indicates we should switch our position from long to short or vice versa). Since we make our trading decisions after the New York market close on each day ${ }^{15}$, it would not be unreasonable to assume only small random variability in the quoted price in the short time ${ }^{16}$ that we take to determine the forecast and place the trade. Furthermore, it is unlikely that historical traders were identifying precisely the same trading opportunities as our forecasting methodology, and thus we would not expect the profitability of the opportunities to decrease due to timing issues $^{17}$. Thus, on average we would expect that, in the long run, we enter into half of the

\footnotetext{
${ }^{15}$ It is important to note that foreign exchange markets are open continuously from Sunday afternoon to Friday afternoon (Eastern time zone). They are closed worldwide from New York close on Friday to New Zealand open on Sunday.

${ }^{16}$ The time needed to compute a single forecast and execute the corresponding trade would realistically be around 30 seconds. Using an automated trading system, this time could be as low as one or two seconds.

${ }^{17}$ If we were not using historical data, and were attempting to generate forecasts for future values of an exchange rate, trade timing can be a significant problem provided we assume other traders are able to obtain the same forecasts as we are.
} 
trades at a slightly less-than-optimal price (resulting in a smaller return) and we enter into the other half at a slightly better-than-optimal price (resulting in a larger return). The overall effect of the timing problem on returns would be zero in the long run.

Transaction costs can potentially have a very large impact on the return of a trading strategy. Neely, Weller \& Dittmar (1997), Neely \& Weller (2003), and Qi \& Wu (2006) argue that the one-way transactions cost for trading a major currency pair is between 2 and 2.5 basis points. Lyons (2001) suggests that for a large institutional trader, a realistic transactions cost would be around 1 to 2 basis points. We would argue that the one-way transactions cost for a large institutional trader at the time of writing is likely 0.5 basis points or less. At times of high liquidity, it is not be uncommon to be able to obtain the same bid and ask prices. Very high frequency intra-minute oscillations in rates also imply that very large traders can often overcome the spread by waiting for the market to move slightly in a favourable direction before placing a trade. Historically, this would not have been as likely. In the early 1990s, prior to the introduction of Electronic Communications Network (ECN) technology, it is much more probable that the one-way transactions costs between large institutional traders was around 2 basis points (or 4 basis points for a complete trade). Consequently, we consider one-way transaction costs of $0.5,1$, and 2 basis points. The return net of transactions costs is then simply:

$$
n e t_{t}=r e t_{t}-2 * T C,
$$

where ret $_{t}$ is the daily $\log$ return defined in section 5 , and $T C$ is the one-way transactions cost. The corresponding net annualized return (NAR) is given by: 


$$
N A R=\left[\prod_{t=T_{0}}^{T_{0}+N-1}\left(1+n e t_{t}\right)\right]^{D / N}-1 .
$$

A simple measure of the possible risk that we might face is the annualized volatility of returns. We define the annualized volatility based on a constant variance random walk as is commonly seen in the finance literature:

$$
A V=\frac{\sigma}{\sqrt{1 / D}},
$$

where $\sigma$ is the sample standard deviation of net $_{t}$. Since this definition of annualized volatility requires that the daily log returns are independent over time, we should consider it to be an approximation of the true annualized volatility.

Two statistics that give valuable information on profitability and risk are the ideal profit ratio and the Sharpe ratio. The ideal profit ratio denotes the fraction of the maximum possible return that is obtained by the trading strategy. The maximum possible return is achieved when the return for every day in the sample is correctly predicted. Thus, the ideal profit ratio is given by:

$$
I R=\sum_{t=T_{0}}^{t=T_{0}+N-1} n e t_{t} / \sum_{t=T_{0}}^{t=T_{0}+N-1}\left(\left|r e t_{t}\right|-2 * T C\right) .
$$

The commonly used Sharpe ratio (see Sharpe (1994) for a recent treatment) measures the average return per unit of risk. The Sharpe ratio is defined as:

$$
S R=\frac{N A R}{A V},
$$

where NAR is the net annualized return, and AV is the annualized volatility as defined above. LeBaron (1999) suggests that for buy and hold strategies on aggregate U.S. stock 
portfolios, Sharpe ratios between 0.3 and 0.4 are common. Similarly, Chang \& Osler (1995) estimate that the Sharpe ratio of the S\&P 500 index was 0.32 between 1974 and 1994.

It is also important to know how often we expect to make a profit and how often we expect to face a loss. Hence, we define the sample probability of a gain and the sample probability of a loss as:

$$
P_{G}=\frac{1}{N} \sum_{t=T_{0}}^{t=T_{0}+N-1} I\left(n e t_{t}\right), \quad P_{L}=\frac{1}{N} \sum_{t=T_{0}}^{t=T_{0}+N-1}\left(1-I\left(n e t_{t}\right)\right), \quad I(x)=\left\{\begin{array}{ll}
1 & \text { if } x \geq 0 \\
0 & \text { if } x<0
\end{array} .\right.
$$

We also define the sample mean gain as:

$$
M_{G}=\sum_{t=T_{0}}^{t=T_{0}+N-1} I_{G}\left(n e t_{t}\right) * n e t_{t} / \sum_{t=T_{0}}^{t=T_{0}+N-1} I_{G}\left(n e t_{t}\right)
$$

We define $M_{L}$ as the sample mean loss in a similar manner.

Additional information about the risk of a trading strategy can be obtained by determining the probability of a sizeable loss. Following Sarantis (2006), we calculate the probability of $10 \% \operatorname{loss}^{18}$ using the method proposed by Dunis \& Williams (2002). Specifically, the probability of loss is given by:

$$
\begin{gathered}
P o L=[(1-P) / P]^{(R / A)}, \\
P=0.5(1+(Z / A)), \quad Z=P_{G} M_{G}+P_{L} M_{L}, \quad A=\left(P_{G} M_{G}^{2}+P_{L} M_{L}^{2}\right)^{1 / 2},
\end{gathered}
$$

where $P o L$ is the probability of loss, $R$ is the maximum level of risk (10\% in our case), and $P_{G}, M_{G}, P_{L}$, and $M_{L}$ are as defined above.

${ }^{18}$ The probability of loss (PoL) is similar to the more commonly known value at risk (VaR) measure. In the case of VaR we fix the probability of a loss, and determine the minimum loss that we would face (which is called the VaR), whereas in the case of PoL we fix the minimum loss (or in other words, we fix our risk tolerance) and determine the probability of this loss occurring. 
Table 8 presents the profitability and risk statistics for forecasts based on the LADBI (KTC) estimator with MAE-optimized samples. For sub-table 8.1, the NAR is identical to the GAR, since there are no transactions costs. For the currency pairs where we have a positive NAR, the Sharpe ratios are very high, with those of EURUSD and EURCHF being greater than one. These Sharpe ratios are significantly higher than the previously mentioned Sharpe ratio of 0.32 for the S\&P 500 index. For the six currency pairs with positive NARs, the ideal profit ratios are between 0.05 and 0.09 . The probability of a $10 \%$ loss is below $5 \%$ for five currency pairs, and below $2.5 \%$ for three currency pairs (GBPUSD, EURUSD, EURCHF).

As we would expect, the NAR, Sharpe ratio, and ideal profit ratio fall and the probability of a $10 \%$ loss increases as the one-way transactions cost increases. In the case of a one basis point one-way transactions cost (Table 8.3), all six currency pairs that initially had positive GARs have positive NARs (with four NARs greater than 5\%), and have Sharpe ratios greater than the S\&P 500 index. Even in the case of a large two basis point one-way transactions cost (Table 8.4), GBPUSD, USDCHF, EURUSD, and EURJPY all have Sharpe ratios greater than the S\&P 500, and USDCHF, EURUSD, and EURJPY have NARs in excess of $5 \%$. While the probability of a $10 \%$ loss is quite large with the two basis point transactions cost, it is still near 10\% for GBPUSD, EURUSD, and EURJPY.

It is important to note that the currency pairs where the NAR and Sharpe ratios remain large when we have large transactions costs are among the most traded currency pairs in the overall foreign exchange market (GBPUSD and EURUSD, in particular). 
Consequently, we believe that the excess returns due to market inefficiencies are very significant, even when we consider the costs of market transactions.

These returns are very similar to those recently obtained in the exchange rate forecasting literature when using either trading signals generated by technical analysis or those produced by time series models. Overall, we obtain gross annual returns from $-1.04 \%$ to $10.40 \%$ over the eight currency pairs that we consider. Given a two basis point one-way transactions cost, we obtain net annual returns over these currency pairs between $-4.91 \%$ and $6.66 \%$. Unfortunately, there are not yet many returns results in the literature for forecasts of EUR-based currencies or USDCAD. GBPUSD, USDCHF, USDJPY and USDDEM appear in most of the existing studies. Over these four currency pairs (by taking EURUSD as the current version of USDDEM) we obtain returns between $6.14 \%$ and $10.40 \%$ with no transactions costs, and returns between $2.67 \%$ and $6.66 \%$ given a two basis point one-way transactions cost.

Several papers have demonstrated significant annual returns using technical analysis rules, even in the presence of substantial transactions costs. Neely, Weller \& Dittmar (1997) obtain annual returns from $1.02 \%$ to $6.05 \%$ given a 2.5 basis point oneway transactions cost using a genetic programming approach to technical analysis rules for four USD-based major currency pairs and two cross pairs. LeBaron (1999) finds $7.00 \%$ to $9.73 \%$ annual returns with zero transactions costs for two USD based majors, using moving average trading rules. Qi \& Wu (2006) obtain annual returns between $1.72 \%$ and $5.72 \%$ out-of-sample, with no transactions costs, for seven currencies versus USD, including GBP, CHF, JPY, and CAD. Neely \& Weller (2003) find annual returns 
of $6.35 \%$ for USDCHF to $9.60 \%$ for USDGBP with a two basis point one-way transactions cost, using intraday technical analysis trading rules.

Both Sarantis (2006) and Dueker \& Neely (2007) have recently found significant annual returns when using time series models that incorporate nonlinearities. Sarantis (2006) obtains returns between $2.61 \%$ for GBPUSD and $28.16 \%$ for USDJPY given a two basis point one-way transactions cost using a Bayesian VAR model with timevarying parameters. Clearly, this model is substantially more sophisticated than the AR(1) model that we employ here, and consequently is able to produce much larger returns in some cases. Notably, in terms of returns, the methodology used in this paper performs slightly better for GBPUSD, while performing substantially worse for USDJPY. The Markov switching model combined with technical analysis trading signals by Dueker \& Neely (2007) produces annual returns between $2.23 \%$ and $6.18 \%$ given an exceptionally large five basis point one-way transactions cost for four USD based major currency pairs. This large transactions cost does not greatly affect the Markov switching model since it only averages 15.5 trades per year over the four USD based currency pairs (GBPUSD, USDCHF, USDJPY, and USDDEM). Conversely, our AR(1) model averages 67.2 trades per year over these four currency pairs, implying that transactions costs have 4.3 times more impact. As a result, we would not expect the returns from the Markov switching model to increase substantially if we lowered the transactions costs to a more realistic level. Thus, we believe that our returns compare quite well with those of Dueker \& Neely (2007), particularly for USDCHF and GBPUSD. 
Similarly, the Sharpe ratios presented in Table 8 compare favourably with those found in the literature. In the case of no transactions costs, the Sharpe ratios that we obtain for currency pairs where returns are positive are between 0.54 and 1.07 . With a two basis point one-way transactions cost, the Sharpe ratios for these currency pairs are between 0.14 and 0.69. Neely, Weller \& Dittmar (1997) find Sharpe ratios between 0.10 and 0.50 with a 2.5 basis point one-way transactions cost. LeBaron (1999) obtains ratios from 0.69 to 1.03 with no transactions costs, while Qi \& Wu (2006) find Sharpe ratios between 0.53 and 0.75 also without transactions costs. Sarantis (2006) obtains Sharpe ratios between 0.33 and 2.37 with a two basis point one-way transactions cost. Dueker \& Neely (2007) find values of the ratio between 0.31 and 0.69 with a five basis point oneway transactions cost.

Overall, we find that both our returns and our Sharpe ratios are very similar to those obtained in the literature when using technical analysis trading rules. Some recent applications of sophisticated time series models have demonstrated larger returns and Sharpe ratios, though our methodology does perform better in some specific cases. In consideration of the extremely simple model that we use, the annual returns produced by the methodology presented here are quite substantial.

\section{Forecast Combinations}

A common method in the forecasting literature for managing model and parameter uncertainty is to take combinations of forecasts. A wide variety of combination methods 
have been suggested (see, for example, Clemen (1989), Diebold \& Lopez (1996), and Timmermann (2006)) and it has been shown that forecast accuracy can be significantly improved by using combinations in many cases. However, forecast combinations have not been widely applied in the context of forecasting exchange rates at high frequencies ${ }^{19}$. We apply several simple combination methods in this section. As Clemen (1989) points out, it is often the case that simple forecast combinations work better than complex combinations.

Suppose we have $J$ forecasts, $f_{T+1, j}$, of the observation at time $T+1$. The linear combination of these $J$ forecasts is formed as:

$$
f_{T+1}^{C}=\sum_{j=1}^{J} w_{j} f_{T+1, j}
$$

where $w_{j}$ is the weight assigned to forecast $j$. We use four different weighting schemes. The first is to simply take equal weights of $1 / J$ for each forecast. In this case, the forecast combination is simply the mean of the $J$ forecasts. Following our optimal estimation sample selection method described in section 5 , we could use estimation sample mean squared error or mean absolute error to obtain the weights. Alternatively, we could use the mean likelihood of the estimated model to produce the weights, where the mean likelihood is $M L=\left(1 / n_{T}\right) \Lambda, \Lambda$ is the estimated likelihood and $n_{T}$ is the number of observations in the estimation sample. In the latter three cases, the weights are obtained using the following:

${ }^{19}$ Billio, Sartore \& Toffano (2000) combine forecasts from nonlinear models with the dollar/mark exchange rate only, for example. 


$$
w_{j}=\frac{W_{j}}{\sum_{j=1}^{J} W_{j}}, \quad W_{j}=\left\{M S E_{j}, M A E_{j}, M L_{j}\right\}
$$

where $M S E_{j}, M A E_{j}$, and $M L_{j}$ denote the estimation sample mean squared error, mean absolute error, and mean likelihood, respectively, for the $j$-th forecast. The method of combination using likelihood-based weights is similar to Bayesian model averaging, which is the Bayesian solution to the model uncertainty problem (see Draper (1995) for a review of the theory). This type of averaging uses the usual Schwartz-Bayes Information Criterion (SBIC) to form the forecast weights. Of course, the SBIC is a likelihood based model selection criterion.

We consider forecast combinations over estimators and over estimation samples. We consider the combination of forecasts from the LAD-BI estimator with those from the MLE-t estimator, and the combination of forecasts from these two estimators with those from the OLS estimator. In both cases we use samples optimized based on estimation sample MAE. We do not consider weighting based on likelihood since the likelihood functions differ across estimators. All of the results in this section are based on the $\Delta s_{t}$ model, as given by equation (1).

Table 9 presents results for forecast combinations over estimators. From these results, we can see that adding information from the MLE-t and OLS estimators does not improve the overall portfolio GAR for any of the combination schemes that we consider. Recall that the portfolio GAR for the LAD-BI specification, with optimal samples selected using MAE, and the kurtosis-based tuning constant (denoted KTC) was 5.19\%, whereas the maximum portfolio GAR that we obtain in Table 9 is $4.17 \%$. However, we 
do see small gains in some individual cases, such as for USDCAD and EURGBP, when we combine across all three estimators. None of the weighting methods appears to dominate over the others, suggesting that the simple forecast mean is likely the best choice.

We examine several different combinations across estimation samples. We use LAD-BI (KTC)-based forecasts for all of the estimation samples that we consider. Pesaran \& Timmermann (2007) suggest that a combination of forecasts from rolling windows is a good approach when there is the possibility of small undetectable structural breaks in the time series. We consider combinations across rolling windows of 500 and 1000 observations, as well as combinations across rolling windows of 100,500 , and 1000 observations. We also consider combinations across estimation samples optimized using MSE and those optimized using MAE. Finally, we look at combinations between rolling estimation windows, expanding estimation windows, and optimal estimation windows.

Table 10 contains results for forecast combinations across estimation samples. In Table 10.1, we see that the maximum portfolio GAR of $3.06 \%$ for combinations based on mean likelihood over the 500 and 1000 observation rolling windows is slightly below the portfolio GARs that we obtained for the individual rolling windows (based on the $\Delta s_{t}$ model) that we presented in Table 3.1. This occurs primarily because the LAD-BI (KTC) based forecasts produce a return smaller than the LAD estimates for the rolling estimation windows. Having mentioned this, we note that in Table 10.2 the best portfolio GAR for the combination of the forecasts from the three rolling estimation windows is 
$3.55 \%$, which is greater than any of the portfolio GARs in Table $3.1^{20}$. This evidence appears to support the suggestion of Pesaran \& Timmerman (2007) that combinations across rolling windows should work well in the presence of small structural changes. However, we should also point out that our optimal estimation sample selection method based on MAE performs about $46 \%$ better with a portfolio GAR of $5.19 \%$.

Table 10.3 contains results for forecast combinations across estimation samples optimized based on MSE and those optimized based on MAE. The results indicate that this is generally unsuccessful, as we do not see improvements in either the portfolio GAR or the GARs for the individual currency pairs. The obvious conclusion is that the forecasts from the LAD-BI estimator using the samples optimized based on MSE do not contribute additional information to the forecasts from the LAD-BI estimator using the samples optimized based on MAE. Table 10.4 presents results for combination of forecasts based on estimates from a 1000 observation rolling window, and those based on an expanding estimation window. The results for this case are very similar to those when we combine across rolling windows. We note that the best portfolio GAR of $3.61 \%$ is slightly larger than that of either individual set of forecasts.

In Table 10.5, we examine combinations between forecasts produced using estimation samples optimized based on MAE and those produced using an expanding estimation window. The results for individual currency pairs are somewhat conflicting.

\footnotetext{
${ }^{20}$ It is unclear, based solely on the results described here, whether the gains are due to the use of the LAD-BI (KTC) estimator or if they are due to using combinations. Earlier results using the plain LAD estimator (not presented here for brevity) indicate that the gains are due to the use of combinations across rolling windows (and expanding windows, where applicable) and are not due entirely to the use of the LAD-BI estimator.
} 
In the case of EURUSD, we see an increase in the GAR by nearly $53 \%$ using mean likelihood weights, whereas for EURGBP, the GAR falls by over $450 \%$. We also see the GAR for GBPUSD decreases somewhat, while that of USDCAD becomes slightly less negative. Overall, the portfolio GARs for all of the weighting schemes remain effectively the same as the portfolio GAR for the LAD-BI (KTC) forecasts on their own. For the results in Table 10.6, we add forecasts based on a rolling window of 1000 observations to the combination scheme. We see that for some of the currency pairs, USDCAD and EURUSD for example, the GAR improves over that of the LAD-BI (KTC) forecasts and for others, such as USDJPY and EURGBP, it worsens. Overall, the portfolio GAR when we use mean likelihood based weights improves a meager $2.1 \%$ to $5.30 \%$.

We do not find significant evidence that forecast combinations can generally produce GARs that improve over those obtained using the forecasts produced by the estimation procedures presented earlier. When we combine forecasts across estimators, we find no improvement in the portfolio GAR, but some individual currency pairs do improve somewhat. In the case of rolling estimation windows, we do find support for combinations in order to alleviate some sample size uncertainty, but the procedure does not perform as well as our estimation sample optimization procedure. One possible reason for this is that we use only three different rolling windows. It is possible that if we used a much larger number of rolling windows, we would see better GAR statistics. In Table 10.6 we see some very slight improvements in the portfolio GAR, and some substantial individual gains and losses. Over the weighting schemes that we consider in 
Table 10, we see that combinations based on mean likelihood perform quite well, though often the simple forecast mean either produces a larger portfolio GAR or one that is only slightly smaller than the other weighting schemes. Furthermore, though we did not mention it in the discussion above, it is also interesting to note that the RMSE and MAE ratios are generally smaller for the forecast combinations than those for the original forecasts. Overall, the evidence on forecast combinations is quite mixed, and it may be that more sophisticated combination techniques would be more successful, though this is often not the case in the forecasting literature.

\section{Conclusions}

We have shown that there exist significant market inefficiencies that allow us to make realistically profitable forecasts for a number of major currency pairs. To uncover these inefficiencies we have used forecasting methodology that is robust to outliers and structural breaks. We found that the LAD estimator produces forecasts that consistently outperform OLS forecasts, regardless of the estimation sample that we use. This suggests that forecasters should think twice before they immediately assume that OLS or other squared-loss-based estimators are the best choice when forecasting financial time series. We have shown that estimators that are robust to outliers can provide important gains when these series display leptokurtosis. Also, we demonstrated that forecasts based on the first differenced exchange rate series can generate larger returns than those based on the $\log$ first differenced series. This is contrary to the popular belief that the log 
transformation should first be applied to financial data in order to obtain a returns series before attempting to produce forecasts.

We developed a nonparametric data-driven estimation sample selection procedure that selects optimal samples based on a general loss function. Using this sample selection procedure, we were able to obtain returns larger than by using a rolling estimation window or an expanding estimation window, both of which are commonly used in the forecasting literature. This is supportive of earlier results on selection of estimation windows in the presence of breaks (Pesaran \& Timmermann, 2005 \& 2007).

We extended the Krasker-Welsch (1982) bounded influence methods to the LAD estimator to produce a two-stage LAD-BI estimation procedure. Using bounded influence tuning constants adjusted for estimation sample kurtosis, we found a substantial increase in gross returns. We then demonstrated that the forecasts based on this methodology display statistically significant evidence of market timing based on the Pesaran-Timmerman (1992) test, and that the corresponding gross returns are also statistically significant based on modified Diebold-Mariano (1995) tests. Furthermore, even in the presence of significant transactions costs, the net returns for a number of currency pairs remain large and positive, and we found that these returns compare favourably with those found in the literature.

While we did confirm an earlier result that combining forecasts based on estimating over several rolling windows does alleviate some estimation sample size uncertainty (Pesaran \& Timmermann, 2007), we did not find that these combinations produced returns greater than those that we obtained using our forecasting methodology. 
Additionally, combinations across forecasts based on rolling windows, expanding windows, and optimal windows only produced a very small improvement in the overall gross return. We do believe that a more detailed examination of possible forecast combinations and weighting schemes is warranted and we leave this for future research. A further possible direction for future research would be to determine if improvements could be made by combining forecasts from proven technical trading rules with those provided by time series models.

Of significant note is that we demonstrated all of the above using only a first-order autoregressive model. It is very possible that more sophisticated models could demonstrate even larger market inefficiencies by applying the data-driven estimation sample selection procedures and bounded influence methods discussed in this paper. The economic implications of the results described above are quite substantial, since these results imply that there are likely significant inefficiencies in foreign exchange markets that should be further explored in the context of both applied forecasting and economic theory. 


\section{Appendix A: Currency Codes}

The following is a list of the ISO 4217 currency codes for all of the currencies that appear in this paper. The official list of all ISO 4217 currency codes can be found on online at: http://www.iso.org/iso/en/prods-services/popstds/currencycodeslist.html.

$\begin{array}{ll}\text { Currency } & \text { ISO } 4217 \text { Alphabetic Code } \\ \text { Canadian Dollar } & \text { CAD } \\ \text { Swiss Franc } & \text { CHF } \\ \text { German Mark } & \text { DEM } \\ \text { Euro } & \text { EUR } \\ \text { Great Britain Pound Sterling } & \text { GBP } \\ \text { Japanese Yen } & \text { JPY } \\ \text { United States Dollar } & \text { USD }\end{array}$

\section{Appendix B: Data}

Daily quote data for the eight currency pairs examined in this paper were obtained from dataHQ, a subsidiary of Australian financial services company Capital Intelligence Limited. Data for the three USD based pairs (USDCHF, USDJPY, and USDCAD) and GBPUSD start on January 2, 1991 and end on October 25, 2006. Data for the four EUR based pairs (EURUSD, EURCHF, EURJPY, and EURGBP) start on January 4, 1999 and end on October 25, 2006. The exchange rate for a given currency pair on a given day is the bid rate for that pair at New York market close (5:00 P.M. EST). 
The interest rates that we used are 3-month London Interbank Offered Rates (LIBOR) obtained directly from the British Bankers' Association ${ }^{21}$. We use 3-month rates since this is the most common LIBOR rate quoted. Provided the term structure of rates is similar across countries, the term of the rate is unimportant since the relevant information is the interest rate differential when determining the return on a foreign exchange transaction. The interest rate series cover the same time periods as the currency data. For days when the BBA did not set an official LIBOR rate (typically due to holidays) but international currency markets were open ${ }^{22}$, we take the closest previous LIBOR rate available.

\section{Appendix C: Likelihood Functions}

This section describes the likelihood functions that can be maximized to obtain estimates of parameters that are equivalent to those produced by the estimators described in section 3. These likelihood functions are for the model based on the first differenced nominal exchange rate $\left(\Delta s_{t}\right)$ given by equation $(1)$

The normal likelihood function for a sample of $n$ observations is given by:

$$
\Lambda\left(\alpha_{0}, \alpha_{1}, \sigma\right)=\prod_{t=1}^{n} \frac{1}{\sigma \sqrt{2 \pi}} \exp \left(\frac{-\left(\Delta s_{t}-\alpha_{0}-\alpha_{1} \Delta s_{t-1}\right)^{2}}{2 \sigma^{2}}\right)
$$

\footnotetext{
${ }^{21}$ The LIBOR rates used in this paper can be found on the BBA website at: http://www.bba.org.uk/bba/jsp/polopoly.jsp?d=141\&a=627

${ }^{22}$ Currency markets are open an average of 258 days per year whereas the BBA sets LIBOR rates for an average of 252 days per year.
} 
where $\sigma$ is the usual scale parameter. The Student-t likelihood function for a sample of $n$ observations is given by:

$$
\Lambda\left(\alpha_{0}, \alpha_{1}, k\right)=\prod_{t=1}^{n} \frac{\Gamma((k+1) / 2)}{\Gamma(k / 2) \sqrt{k \pi}}\left(1+\frac{\left(\Delta s_{t}-\alpha_{0}-\alpha_{1} \Delta s_{t-1}\right)^{2}}{k}\right)^{-(k+1) / 2},
$$

where $\Gamma(x)$ is the gamma function and $k$ is the number of degrees of freedom. The Laplace likelihood function for a sample of $n$ observations is given by:

$$
\Lambda\left(\alpha_{0}, \alpha_{1}, b\right)=\prod_{t=1}^{n} \frac{1}{2 b} \exp \left(\frac{-\left|\Delta s_{t}-\alpha_{0}-\alpha_{1} \Delta s_{t-1}\right|}{b}\right)
$$

where $b$ is a scale parameter.

\section{Appendix D: Bootstrap Estimation of Krasker-Welsch Tuning Constant}

We employ a bootstrap procedure to estimate the tuning constant needed to form Krasker-Welsch bounded influence samples. The bootstrap procedure that we use is similar to a nonparametric block bootstrap where the block size is random.

For each currency pair we implement the following procedure. We first select the estimation sample for the bootstrap, which consists of all observations where $t<T_{0}$. For each bootstrap replication, we randomly select 20 block subsamples, each of a random size between 50 and 250 observations, from the estimation sample. For each of the 20 subsamples, we do the following:

1. Calculate and store the sample kurtosis. 
2. Form the DFFITS series using equation (4). Note that we cannot simply do this for the full estimation sample, since the values of DFFITS effectively depend on the OLS estimate over the sample.

3. For each value of $\mathrm{c}$ between 1.45 and 10.0 (using a step size of 0.05 ) construct a Krasker-Welsch bounded influence sample containing observations that satisfy the inequality in $(5)^{23}$. For each of the bounded influence samples, calculate a one-step-ahead LAD forecast for the out-of-sample observation directly following the last observation in the subsample.

4. Calculate and store the return produced by following the trading signal generated from the forecast in step 3.

Next, we calculate and store the mean kurtosis over the 20 subsamples. We calculate the mean return over the 20 subsamples for each value of $c$. The optimal value of $c$ over the 20 subsamples is the one that produces the largest out-of-sample return.

After 2000 bootstrap replications, we have 2000 optimal tuning constant and mean kurtosis observations. The mean optimal tuning constant over the 2000 observations is the bootstrap estimate of the fixed tuning constant for the given currency. An OLSestimated regression of the tuning constant on the mean kurtosis and a constant gives the linear relationship between the estimation sample kurtosis and the tuning constant.

\footnotetext{
${ }^{23}$ Note that the lower bound of the grid search is set to 1.45 since the lowest possible value of the tuning constant is the square root of the number of parameters in the estimated model (Krasker \& Welsch, 1982). In all cases, the number of parameters in the model is equal to two (one each for the constant and the lagged dependent variable)
} 


\section{Appendix E: Bootstrapping of Diebold-Mariano Statistics}

Following the suggestion of Clark and McCracken (2005), we use the wild bootstrap procedure recommended by Goncalves and Kilian (2004) to produce approximate empirical distributions of the Diebold-Mariano statistic (also called MSE-T or OOS-t). This method has the advantage of preserving (to some extent) the time-dependency of the data in light of the possibility of conditional heteroskedasticity in the forecast errors.

The exact algorithm that we use to produce the empirical distributions of the Diebold-Mariano statistic is as follows for the first differenced nominal exchange rate $\operatorname{series}\left(\Delta s_{t}\right)$ :

1. Estimate the (null) random walk model with drift, $\Delta s_{t}=\mu+\varepsilon_{t}$, over the full sample, $t=1, \ldots, T_{F}$ and save the estimated mean parameter as $\hat{\mu}$ and the residuals as $\hat{\varepsilon}_{t}$.

2. Draw an independent and identically distributed $T_{F} \times 1$ vector $z$ from the standard normal distribution.

3. Generate a simulated series $\Delta s_{t}^{*}$ as $\Delta s_{t}^{*}=\hat{\mu}+\hat{\varepsilon}_{t} z_{t}$.

4. Generate $N$ forecasts from both the random walk model, and the forecasting model under consideration, by estimating the models over the appropriate subsamples of the simulated series $\Delta s_{t}^{*}$. Recall that both models are re-estimated for a forecast of the value at time $T+1$ using information up to time $T$, so this step implies $N$ total estimations of both models using the simulated series. 
5. Calculate and save the Diebold-Mariano statistic based on the forecasts generated in step 4. We also save the GAR of the model under consideration for the returns test.

6. Repeat steps two through five 1000 times. The Diebold-Mariano statistics generated in step 5 make up the empirical distribution of this statistic under the null hypothesis. 


\section{References}

Baillie, R.T. and T. Bollerslev (1989). "The Message in Daily Exchange Rates: A Conditional-Variance Tale.” Journal of Business \& Economic Statistics 7, 60-68.

Baillie, R.T. and T. Bollerslev (1991). "Intra-Day and Inter-Market Volatility in Foreign Exchange Rates.” Review of Economic Studies 58, 565-585.

Baillie, R.T., T. Bollerslev and H.O. Mikkelsen (1996). "Fractionally integrated generalized autoregressive conditional heteroskedasticity." Journal of Econometrics 74, 3-30.

Bank for International Settlements (2005). Triennial Central Bank Survey: Foreign exchange and derivatives market activity in 2004. Basel, Switzerland: BIS Press \& Communications.

Belsley, D.A., E. Kuh and R.E. Welsch (1980). Regression Diagnostics: Identifying Influential Data and Sources of Collinearity. New York: John Wiley \& Sons.

Berkowitz, J. and L. Giorgianni (2001). “Long-Horizon Exchange Rate Predictability?" Review of Economics and Statistics 83, 81-91.

Billio, M.D., D. Sartore and C. Toffano (2000). "Combining forecasts: some results on exchange and interest rates." European Journal of Finance 6, 126-145.

Boero, G. and E. Marrocu (2002). "The Performance of Non-linear Exchange Rate Models: a Forecasting Comparison." Journal of Forecasting 21, 513-542.

Boothe, P. and D. Glassman (1987a). "Comparing Exchange Rate Forecasting Models: Accuracy versus Profitability." International Journal of Forecasting 3, 65-79. 
Boothe, P. and D. Glassman (1987b). "The Statistical Distribution of Exchange Rates: Empirical Evidence and Economic Interpretations.” Journal of International Economics 22, 297-319.

Brooks, C. (1996). “Testing for non-linearity in daily sterling exchange rates." Applied Financial Economics 6, 307-317.

Brooks, C. (1997). "Linear and Non-linear (Non-)Forecastability of High-frequency Exchange Rates." Journal of Forecasting 16, 125-145.

Brown, R.L., J. Durbin and J.M. Evans (1975). "Techniques for testing the constancy of regression relationships over time." Journal of the Royal Statistical Society, Series $B 36,149-192$.

Chang, P.H.K. and C.L. Osler (1999). "Methodical Madness: Technical Analysis and the Irrationality of Exchange-Rate Forecasts." Economic Journal 109, 636-661.

Cheung, Y.W., M.D. Chinn and A.G. Pascual (2005). "Empirical exchange rate models of the nineties: Are any fit to survive?" Journal of International Money and Finance 24, 1150-1175.

Chinn, M. and R. Meese (1995). "Banking on Currency Forecasts: How Predictable is Change in Money?" Journal of International Economics 38, 161-178.

Clemen, R.T. (1989). "Combining Forecasts: A Review and Annotated Bibliography." International Journal of Forecasting 5, 559-581.

Clements, M.P. and Smith, J. (2001). "Evaluating forecasts from SETAR models of exchange rates." Journal of International Money and Finance 20, 133-148. 
Clark, T.E. and M.W. McCracken (2001). “Tests of equal forecast accuracy and encompassing for nested models." Journal of Econometrics 105, 85-110.

Clark, T.E. and M.W. McCracken (2005a). "Evaluating Direct Multistep Forecasts." Econometric Reviews 24, 369-404.

Clark, T.E. and M.W. McCracken (2005b). "The power of tests of predictive ability in the presence of structural breaks." Journal of Econometrics 124, 1-31.

Dewachter, H. (2001). "Can Markov switching models replicate chartist profits in the foreign exchange market?" Journal of International Money and Finance 20, 25-41.

Diebold, F.X. and J. Lopez (1996). "Forecast Evaluation and Combination." in G.S. Maddala and C.R. Rao, eds., Handbook of Statistics, Volume 14. Elsevier: Amsterdam.

Diebold, F.X. and R.S. Mariano (1995). "Comparing Predictive Accuracy." Journal of Business \& Economic Statistics 13, 253-263.

Diebold, F.X. and J.A. Nason (1990). “Nonparametric Exchange Rate Prediction?” Journal of International Economics 28, 315-332.

Draper, D. (1995). “Assessment and Propagation of Model Uncertainty." Journal of the Royal Statistical Society B 57, 45-97.

Dueker, M. and C.J. Neely (2007). “Can Markov switching models predict excess foreign exchange returns?" Journal of Banking \& Finance 31, 279-296.

Dunis, C., and M. Williams (2002). "Modeling and trading the EURO/USD exchange rate: Do neural network models perform better?" in C. Dunis, P. Naim, and L. 
Laws, eds., Applied Quantitative Methods for Trading and Investment. John Wiley \& Sons: Chichester, U.K.

Gençay, R. (1999). "Linear, non-linear and essential foreign exchange rate prediction with simple technical trading rules." Journal of International Economics 47, 91107.

Goncalves, S. and L. Kilian (2004). "Bootstrapping autoregressions with conditional heteroskedasticity of unknown form." Journal of Econometrics 123, 89-120.

Harvey, D., S. Leybourne, and P. Newbold (1997). "Testing the equality of prediction mean squared errors." International Journal of Forecasting 13, 281-291.

Harvey, D., S. Leybourne, and P. Newbold (1999). "Forecast Evaluation Tests in the Presence of ARCH." Journal of Forecasting 18, 435-445.

Henriksson, R.D. and R.C. Merton (1981). “On Market Timing and Investment Performance. II. Statistical Procedures for Evaluating Forecasting Skills.” Journal of Business 54, 513-533.

Hsieh, D.A. (1988). "The Statistical Properties of Daily Foreign Exchange Rates: 19741983." Journal of International Economics 24, 129-145.

Hseih, D.A. (1989). "Modeling Heteroscedasticity in Daily Foreign-Exchange Rates." Journal of Business \& Economic Statistics 7, 307-317.

Inoue, A. and L. Kilian (2004). "In-Sample or Out-of-Sample Tests of Predictability: Which One Should We Use?" Econometric Reviews 23, 371-402. 
Judge, G., R.C. Hill, W.E. Griffiths, H. Lutkepohl, and T. Lee (1988). Introduction to the Theory and Practice of Econometrics, Second edition, John Wiley \& Sons: New York.

Kelly, G.E. (1992). "Robust Regression Estimators - The Choice of Tuning Constants." The Statistician 41, 303-314.

Kelly, G.E. (1996). “Adaptive Choice of Tuning Constant for Robust Regression Estimators." The Statistician 45, 35-40.

Kilian, L. (1999). "Exchange Rates and Monetary Fundamentals: What do we Learn from Long-Horizon Regressions?" Journal of Applied Econometrics 14, 491-510.

Kilian, L. and M.P. Taylor (2003). "Why is it so difficult to beat the random walk forecast of exchange rates." Journal of International Economics 60, 85-107.

Kräger H. and P. Kugler (1993). "Non-linearities in foreign exchange markets: a different perspective." Journal of International Money and Finance 12, 195-208.

Krasker, W.S., E. Kuh, and R.E. Welsch (1983). "Estimation for Dirty Data and Flawed Models." in Z. Griliches and M.D. Intriligator, eds., Handbook of Econometrics, Volume 1, North-Holland: Amsterdam.

Krasker, W.S. and R.E. Welsch (1982). "Efficient Bounded Influence Regression Estimation." Journal of the American Statistical Association 77, 595-604.

Lange, K.L., R.J.A. Little, and J.M.G. Taylor (1989). "Robust Statistical Modeling using the t-distribution." Journal of the American Statistical Association 84, 881-896.

LeBaron, B. (1992). “Do Moving Average Trading Rule Results Imply Nonlinearities in Foreign Exchange Markets?” University of Wisconsin-Madison, Working Paper. 
LeBaron, B. (1999). "Technical trading rule profitability and foreign exchange intervention." Journal of International Economics 49, 125-143.

Levich, R.M. and L.R. Thomas (1993). "The significance of technical trading-rule profits in the foreign exchange market: a bootstrap approach." Journal of International Money and Finance 12, 451-474.

Lyons, R.K. (2001). The Microstructure Approach to Exchange Rates. The MIT Press: Cambridge, Massachusetts.

Mark, N.C. (1995). "Exchange Rates and Fundamentals: Evidence on Long-horizon Predictability." American Economic Review 85, 201-218.

Mark, N.C and D. Sul (2001). "Nominal exchange rates and monetary fundamentals: Evidence from a small post-Bretton Woods panel." Journal of International Economics 53, 29-52.

Martin, R.D. (1979). "Robust Estimation for Time Series Autoregressions." in R.L. Launer and G. Wilkinson, eds., Robustness in Statistics. Academic Press: New York.

Meade, N. (2002). "A comparison of the accuracy of short term foreign exchange forecasting methods." International Journal of Forecasting 18, 67-83.

Meese R.A. and K. Rogoff (1983). "Empirical exchange rate models of the seventies: do they fit out of sample?" Journal of International Economics 14, 3-24.

Meese, R.A. and A.K. Rose (1991). “An Empirical Assessment of Non-Linearities in Models of Exchange Rate Determination." Review of Economic Studies 58, 603619. 
McCracken, M.W. (2000). “Robust out-of-sample inference.” Journal of Econometrics $99,195-223$.

McCracken, M.W. (2004a). “Asymptotics for Out of Sample Tests of Granger Causality." University of Missouri-Columbia Working Paper (forthcoming in the Journal of Econometrics).

McCracken, M.W. (2004b). "Parameter estimation and test of equal forecast accuracy between non-nested models." International Journal of Forecasting 20, 503-514.

Nag, A.K. and A. Mitra (2002). "Forecasting Daily Foreign Exchange Rates Using Genetically Optimized Neural Networks.” Journal of Forecasting 21, 501-511.

Neely, C.J. (1997). “Technical Analysis in the Foreign Exchange Market: A Layman's Guide." Federal Reserve Bank of St. Louis Review, September/October 1997, 2338.

Neely, C.J. and P.A. Weller (2003). "Intraday technical trading in the foreign exchange market." Journal of International Money and Finance 22, 223-237.

Neely, C.J., P.A. Weller and R. Dittmar (1997). "Is Technical Analysis in the Foreign Exchange Market Profitable? A Genetic Programming Approach." Journal of Financial and Quantitative Analysis 32, 405-426.

Okunev, J. and D. White (2003). “Do Momentum-Based Strategies Still Work in Foreign Currency Markets?” Journal of Financial and Quantitative Analysis 38, 425-447.

Olson, D. (2004). “Have trading rule profits in the currency markets declined over time?" Journal of Banking \& Finance 28, 85-105. 
Pesaran, M.H. and A. Timmermann (1992). "A Simple Nonparametric Test of Predictive Performance." Journal of Business \& Economic Statistics 10, 461-465.

Pesaran, M.H. and A. Timmermann (1994). "A generalization of the nonparametric Henriksson-Merton test of market timing." Economics Letters 44, 1-7.

Pesaran, M.H. and A. Timmermann (2002). "Market timing and return prediction under model instability." Journal of Empirical Finance 9, 495-510.

Pesaran, M.H. and A. Timmermann (2005). "Small sample properties of forecasts from autoregressive models under structural breaks." Journal of Econometrics 129, 183217.

Pesaran, M.H. and A. Timmermann (2007). "Selection of estimation window in the presence of breaks." Journal of Econometrics 137, 134-161.

Peters S.C., A.M. Samarov and R.E. Welsch (1981). "Computational Procedures for Bounded-Influence and Robust Regression." Technical Report No. 30, Massachusetts Institute of Technology Center for Computational Research in Economics and Management Science.

Qi, M. and Y. Wu (2006). "Technical Trading-Rule Profitability, Data Snooping, and Reality Check: Evidence from the Foreign Exchange Market." Journal of Money, Credit, and Banking 38, 2135-2158.

Sarantis, N. (2006). "On the short-term predictability of exchange rates: A BVAR timevarying parameters approach." Journal of Banking \& Finance 30, 2257-2279.

Sweeny, R.J. (1986). "Beating the Foreign Exchange Market." Journal of Finance 41, 163-182. 
Taylor, S.J. (1992). “Rewards Available to Currency Futures Speculators: Compensation for Risk or Evidence of Inefficient Pricing?’ Economic Record 68 (Supplement), $105-116$.

Taylor, S.J. (1994). “Trading Futures Using a Channel Rule: A Study of the Predictive Power of Technical Analysis with Currency Examples." Journal of Futures Markets 14, 215-235.

Timmermann, A. (2006). "Forecast Combinations." in G. Elliott, C.W.J. Granger, and A. Timmerman, eds., Handbook of Economic Forecasting, Elsevier: Amsterdam.

Warwick, J. (2005). “A data-based method for selecting tuning parameters in minimum distance estimators." Computational Statistics \& Data Analysis 48, 571-585.

Warwick, J. and M.C. Jones (2005). “Choosing a robustness tuning parameter.” Journal of Statistical Computation and Simulation 75, 581-588. 
Table 1.1

Summary statistics

First differenced nominal exchange rate $\left(\Delta s_{t}\right)$

\begin{tabular}{l|r|r|r|r} 
& GBPUSD & USDCHF & USDJPY & USDCAD \\
\hline Mean & -0.000017 & 0.000000 & -0.003749 & -0.000008 \\
Standard Deviation & 0.009644 & 0.010140 & 0.805620 & 0.005059 \\
Skewness & -0.281420 & -0.112790 & -0.898360 & -0.040449 \\
Kurtosis & 3.832400 & 1.510960 & 8.981570 & 1.755990 \\
Minimum & -0.080000 & -0.050200 & -9.800000 & -0.022400 \\
$25^{\text {th }}$ Percentile & -0.005100 & -0.005575 & -0.417500 & -0.002700 \\
Median & 0.000100 & 0.000000 & 0.010010 & 0.000000 \\
$75^{\text {th }}$ Percentile & 0.005200 & 0.006000 & 0.440000 & 0.002800 \\
Maximum & 0.055800 & 0.049500 & 4.740010 & 0.023600 \\
\hline
\end{tabular}

\begin{tabular}{l|r|r|r|r} 
& EURUSD & EURCHF & EURJPY & EURGBP \\
\hline Mean & 0.000038 & -0.000012 & 0.008771 & -0.000020 \\
Standard Deviation & 0.006874 & 0.003517 & 0.843940 & 0.003093 \\
Skewness & -0.031675 & -0.526840 & -0.044639 & 0.104020 \\
Kurtosis & 0.737230 & 4.060390 & 2.037120 & 1.044150 \\
Minimum & -0.034600 & -0.026000 & -3.820010 & -0.012000 \\
$25^{\text {th }}$ Percentile & -0.004200 & -0.001900 & -0.520000 & -0.001900 \\
Median & 0.000000 & 0.000100 & 0.049992 & -0.000100 \\
$75^{\text {th }}$ Percentile & 0.004200 & 0.001900 & 0.509990 & 0.001800 \\
Maximum & 0.023300 & 0.019600 & 5.029990 & 0.012800 \\
\hline
\end{tabular}


Table 1.2

Summary statistics

First differenced natural log nominal exchange rate $\left(\Delta l s_{t}\right)$

\begin{tabular}{l|r|r|r|r} 
& GBPUSD & USDCHF & USDJPY & USDCAD \\
\hline Mean & -0.000009 & 0.000000 & -0.000030 & -0.000007 \\
Standard Deviation & 0.005816 & 0.007184 & 0.006918 & 0.003716 \\
Skewness & -0.190070 & -0.114260 & -0.720030 & -0.034335 \\
Kurtosis & 3.230580 & 1.714760 & 7.160440 & 1.852350 \\
Minimum & -0.043963 & -0.035216 & -0.078109 & -0.016971 \\
$25^{\text {th }}$ Percentile & -0.003153 & -0.003989 & -0.003612 & -0.002007 \\
Median & 0.000055 & 0.000000 & 0.000108 & 0.000000 \\
$75^{\text {th }}$ Percentile & 0.003185 & 0.004181 & 0.003858 & 0.002076 \\
Maximum & 0.032334 & 0.037979 & 0.040968 & 0.017309 \\
\hline
\end{tabular}

\begin{tabular}{l|r|r|r|r} 
& EURUSD & EURCHF & EURJPY & EURGBP \\
\hline Mean & 0.000031 & -0.000007 & 0.000062 & -0.000029 \\
Standard Deviation & 0.006413 & 0.002298 & 0.007141 & 0.004784 \\
Skewness & -0.010414 & -0.533860 & -0.036596 & 0.089914 \\
Kurtosis & 0.726260 & 4.468110 & 2.521530 & 1.263080 \\
Minimum & -0.028388 & -0.017837 & -0.033005 & -0.020405 \\
$25^{\text {th }}$ Percentile & -0.003973 & -0.001240 & -0.004222 & -0.002853 \\
Median & 0.000000 & 0.000064 & 0.000361 & -0.000146 \\
$75^{\text {th }}$ Percentile & 0.003859 & 0.001260 & 0.004118 & 0.002773 \\
Maximum & 0.023877 & 0.013476 & 0.041649 & 0.020868 \\
\hline
\end{tabular}


Table 2

Benchmark forecasts

\begin{tabular}{ll|c|c|c|c} 
& & GBPUSD & USDCHF & USDJPY & USDCAD \\
\hline$\Delta s_{t}$ & RMSE & 0.008316 & 0.009709 & 0.819600 & 0.005510 \\
Model & MAE & 0.006237 & 0.007227 & 0.586345 & 0.004154 \\
& PC & $49.73 \%$ & $49.67 \%$ & $48.40 \%$ & $50.07 \%$ \\
& GAR & $-3.06 \%$ & $-4.89 \%$ & $-9.36 \%$ & $-2.26 \%$ \\
\hline$\Delta l s_{t}$ & RMSE & 0.005032 & 0.006859 & 0.007052 & 0.004010 \\
Model & MAE & 0.003797 & 0.005131 & 0.005094 & 0.003002 \\
& PC & $49.73 \%$ & $49.67 \%$ & $48.40 \%$ & $50.07 \%$ \\
& GAR & $-3.06 \%$ & $-4.89 \%$ & $-9.36 \%$ & $-2.26 \%$
\end{tabular}

\begin{tabular}{ll|c|c|c|c|c} 
& & EURUSD & EURCHF & EURJPY & EURGBP & Portfolio \\
\hline$\Delta s_{t}$ & RMSE & 0.007322 & 0.003313 & 0.735950 & 0.002737 & \\
Model & MAE & 0.005609 & 0.002515 & 0.567112 & 0.002089 & \\
& PC & $48.70 \%$ & $47.60 \%$ & $52.30 \%$ & $51.80 \%$ & $49.78 \%$ \\
& GAR & $-8.87 \%$ & $-3.65 \%$ & $-1.61 \%$ & $-1.79 \%$ & $-4.44 \%$ \\
\hline$\Delta s_{t}$ & RMSE & 0.006060 & 0.002152 & 0.005465 & 0.003996 & \\
Model & MAE & 0.004635 & 0.001632 & 0.004188 & 0.003053 & \\
& PC & $48.70 \%$ & $47.60 \%$ & $52.30 \%$ & $51.80 \%$ & $49.78 \%$ \\
& GAR & $-8.87 \%$ & $-3.65 \%$ & $-1.61 \%$ & $-1.79 \%$ & $-4.44 \%$
\end{tabular}

Benchmark model is a random walk with drift estimated by OLS over an expanding window. RMSE is root mean squared error, MAE is mean absolute error, PC is the percentage of forecasts that correctly predict the direction of change, and GAR is the gross annualized return. The "Portfolio" column contains averages over all eight currency pairs. See section 6 for complete definitions of statistics. 
Table 3.1.1

Forecasts based on rolling window

First differenced nominal exchange rate $\left(\Delta s_{t}\right)$

Window size of $n_{T}=100$

\begin{tabular}{ll|c|c|c|c} 
& & GBPUSD & USDCHF & USDJPY & USDCAD \\
\hline \multirow{2}{*}{ OLS } & RMSE & 1.0076 & 1.0104 & 1.0117 & 1.0078 \\
& MAE & 1.0039 & 1.0075 & 1.0094 & 1.0060 \\
& PC & $51.90 \%$ & $51.57 \%$ & $50.60 \%$ & $51.23 \%$ \\
& GAR & $1.49 \%$ & $-1.08 \%$ & $-1.54 \%$ & $1.76 \%$ \\
\hline \multirow{2}{*}{ MLE-t } & RMSE & 1.0087 & 1.0096 & 1.0143 & 1.0074 \\
& MAE & 1.0050 & 1.0051 & 1.0084 & 1.0055 \\
& PC & $51.83 \%$ & $51.97 \%$ & $51.80 \%$ & $51.27 \%$ \\
& GAR & $0.46 \%$ & $-1.21 \%$ & $1.74 \%$ & $2.03 \%$ \\
\hline \multirow{2}{*}{ LAD } & RMSE & 1.0131 & 1.0096 & 1.0167 & 1.0147 \\
& MAE & 1.0091 & 1.0061 & 1.0100 & 1.0125 \\
& PC & $51.90 \%$ & $51.97 \%$ & $52.73 \%$ & $50.73 \%$ \\
& GAR & $1.77 \%$ & $-1.47 \%$ & $4.96 \%$ & $1.76 \%$
\end{tabular}

\begin{tabular}{ll|c|c|c|c|c} 
& & EURUSD & EURCHF & EURJPY & EURGBP & Portfolio \\
\hline \multirow{2}{*}{ OLS } & RMSE & 1.0067 & 1.0112 & 1.0103 & 1.0121 & \\
& MAE & 1.0032 & 1.0068 & 1.0081 & 1.0105 & \\
& PC & $52.80 \%$ & $48.70 \%$ & $51.20 \%$ & $53.00 \%$ & $51.38 \%$ \\
& GAR & $3.79 \%$ & $-2.94 \%$ & $-0.86 \%$ & $-1.89 \%$ & $-0.16 \%$ \\
\hline \multirow{2}{*}{ MLE-t } & RMSE & 1.0074 & 1.0097 & 1.0089 & 1.0128 & \\
& MAE & 1.0045 & 1.0036 & 1.0050 & 1.0105 & \\
& PC & $53.10 \%$ & $51.10 \%$ & $50.40 \%$ & $52.70 \%$ & $51.77 \%$ \\
& GAR & $3.31 \%$ & $0.10 \%$ & $-2.40 \%$ & $-1.17 \%$ & $0.36 \%$ \\
\hline \multirow{2}{*}{ LAD } & RMSE & 1.0071 & 1.0075 & 1.0154 & 1.0190 & \\
& MAE & 1.0059 & 1.0012 & 1.0097 & 1.0187 & \\
& PC & $52.20 \%$ & $54.00 \%$ & $51.80 \%$ & $51.10 \%$ & $52.05 \%$ \\
& GAR & $2.44 \%$ & $2.56 \%$ & $-2.07 \%$ & $-0.99 \%$ & $1.12 \%$
\end{tabular}

RMSE and MAE are root mean squared error and mean absolute error, respectively, both relative to the benchmark results presented in Table 2, PC is the percentage of forecasts that correctly predict the direction of change, and GAR is the gross annualized return. The "Portfolio" column contains averages over all eight currency pairs. See section 6 for complete definitions. 
Table 3.1.2

Forecasts based on rolling window

First differenced nominal exchange rate $\left(\Delta s_{t}\right)$

Window size of $n_{T}=\mathbf{5 0 0}$

\begin{tabular}{ll|c|c|c|c} 
& & GBPUSD & USDCHF & USDJPY & USDCAD \\
\hline \multirow{2}{*}{ OLS } & RMSE & 1.0008 & 1.0003 & 1.0031 & 1.0002 \\
& MAE & 0.9998 & 0.9974 & 0.9990 & 1.0000 \\
& PC & $52.40 \%$ & $53.37 \%$ & $52.10 \%$ & $50.87 \%$ \\
& GAR & $2.71 \%$ & $2.59 \%$ & $1.06 \%$ & $0.52 \%$ \\
\hline \multirow{2}{*}{ MLE-t } & RMSE & 1.0010 & 1.0001 & 1.0058 & 0.9998 \\
& MAE & 0.9997 & 0.9954 & 0.9990 & 1.0000 \\
& PC & $51.97 \%$ & $53.97 \%$ & $51.50 \%$ & $50.67 \%$ \\
& GAR & $3.07 \%$ & $3.85 \%$ & $-0.78 \%$ & $0.95 \%$ \\
\hline \multirow{2}{*}{ LAD } & RMSE & 1.0016 & 1.0013 & 1.0076 & 1.0002 \\
& MAE & 0.9994 & 0.9958 & 1.0019 & 1.0010 \\
& PC & $51.83 \%$ & $54.50 \%$ & $51.20 \%$ & $51.73 \%$ \\
& GAR & $3.00 \%$ & $5.33 \%$ & $-1.03 \%$ & $2.18 \%$
\end{tabular}

\begin{tabular}{ll|c|c|c|c|c} 
& & EURUSD & EURCHF & EURJPY & EURGBP & Portfolio \\
\hline \multirow{2}{*}{ OLS } & RMSE & 0.9990 & 1.0003 & 1.0023 & 1.0015 & \\
& MAE & 0.9961 & 0.9960 & 1.0003 & 1.0034 & \\
& PC & $53.20 \%$ & $52.00 \%$ & $52.60 \%$ & $50.40 \%$ & $52.12 \%$ \\
& GAR & $7.47 \%$ & $0.35 \%$ & $2.22 \%$ & $0.42 \%$ & $2.17 \%$ \\
\hline \multirow{2}{*}{ MLE-t } & RMSE & 0.9993 & 1.0012 & 1.0022 & 1.0011 & \\
& MAE & 0.9966 & 0.9960 & 0.9993 & 1.0029 & \\
& PC & $53.00 \%$ & $53.10 \%$ & $53.30 \%$ & $50.50 \%$ & $52.25 \%$ \\
& GAR & $4.38 \%$ & $1.84 \%$ & $4.50 \%$ & $-1.78 \%$ & $2.00 \%$ \\
\hline \multirow{2}{*}{ LAD } & RMSE & 1.0000 & 1.0027 & 1.0031 & 1.0026 & \\
& MAE & 0.9973 & 0.9964 & 0.9977 & 1.0034 & \\
& PC & $52.70 \%$ & $55.20 \%$ & $54.40 \%$ & $51.90 \%$ & $52.93 \%$ \\
& GAR & $5.27 \%$ & $4.53 \%$ & $5.48 \%$ & $2.96 \%$ & $3.47 \%$
\end{tabular}

See notes to Table 3.1.1 for details on cell entries. 
Table 3.1.3

Forecasts based on rolling window

First differenced nominal exchange rate $\left(\Delta s_{t}\right)$

Window size of $n_{T}=1000$

\begin{tabular}{ll|c|c|c|c} 
& & GBPUSD & USDCHF & USDJPY & USDCAD \\
\hline \multirow{2}{*}{ OLS } & RMSE & 1.0030 & 0.9992 & 1.0021 & 0.9991 \\
& MAE & 1.0027 & 0.9982 & 1.0008 & 0.9993 \\
& PC & $49.67 \%$ & $52.43 \%$ & $48.43 \%$ & $52.03 \%$ \\
& GAR & $-3.37 \%$ & $1.63 \%$ & $-8.65 \%$ & $3.48 \%$ \\
\hline \multirow{2}{*}{ MLE-t } & RMSE & 1.0025 & 0.9994 & 1.0047 & 0.9987 \\
& MAE & 1.0019 & 0.9965 & 1.0005 & 0.9990 \\
& PC & $50.53 \%$ & $53.13 \%$ & $50.33 \%$ & $51.97 \%$ \\
& GAR & $-1.98 \%$ & $4.39 \%$ & $-0.68 \%$ & $3.67 \%$ \\
\hline \multirow{2}{*}{ LAD } & RMSE & 1.0025 & 1.0000 & 1.0042 & 0.9989 \\
& MAE & 1.0018 & 0.9963 & 1.0008 & 0.9995 \\
& PC & $51.53 \%$ & $53.80 \%$ & $50.30 \%$ & $51.97 \%$ \\
& GAR & $0.81 \%$ & $4.84 \%$ & $0.47 \%$ & $4.38 \%$
\end{tabular}

\begin{tabular}{ll|c|c|c|c|c} 
& & EURUSD & EURCHF & EURJPY & EURGBP & Portfolio \\
\hline OLS & RMSE & 0.9993 & 1.0000 & 1.0006 & 1.0015 & \\
& MAE & 0.9968 & 0.9976 & 0.9985 & 1.0043 & \\
& PC & $54.00 \%$ & $51.20 \%$ & $53.00 \%$ & $48.00 \%$ & $51.10 \%$ \\
& GAR & $7.38 \%$ & $-0.58 \%$ & $1.48 \%$ & $-4.38 \%$ & $-0.38 \%$ \\
\hline \multirow{2}{*}{ MLE-t } & RMSE & 0.9997 & 1.0000 & 1.0003 & 1.0018 & \\
& MAE & 0.9975 & 0.9960 & 0.9972 & 1.0048 & \\
& PC & $54.30 \%$ & $51.90 \%$ & $54.70 \%$ & $48.70 \%$ & $51.95 \%$ \\
& GAR & $7.88 \%$ & $-0.30 \%$ & $5.89 \%$ & $-3.10 \%$ & $1.97 \%$ \\
\hline \multirow{2}{*}{ LAD } & RMSE & 0.9999 & 1.0003 & 1.0010 & 1.0018 & \\
& MAE & 0.9968 & 0.9960 & 0.9958 & 1.0057 & \\
& PC & $54.70 \%$ & $53.70 \%$ & $55.10 \%$ & $49.40 \%$ & $52.56 \%$ \\
& GAR & $10.38 \%$ & $1.78 \%$ & $7.53 \%$ & $-2.57 \%$ & $3.45 \%$
\end{tabular}

See notes to Table 3.1.1 for details on cell entries. 
Table 3.2.1

Forecasts based on rolling window

First differenced natural log nominal exchange rate $\left(\Delta l s_{t}\right)$

Window size of $n_{T}=100$

\begin{tabular}{ll|c|c|c|c} 
& & GBPUSD & USDCHF & USDJPY & USDCAD \\
\hline OLS & RMSE & 1.0078 & 1.0102 & 1.0113 & 1.0072 \\
& MAE & 1.0042 & 1.0076 & 1.0088 & 1.0057 \\
& PC & $51.87 \%$ & $51.37 \%$ & $50.63 \%$ & $51.17 \%$ \\
& GAR & $1.30 \%$ & $-1.66 \%$ & $-1.43 \%$ & $1.56 \%$ \\
\hline \multirow{2}{*}{ MLE-t } & RMSE & 1.0087 & 1.0093 & 1.0145 & 1.0075 \\
& MAE & 1.0053 & 1.0051 & 1.0082 & 1.0053 \\
& PC & $52.10 \%$ & $52.10 \%$ & $51.57 \%$ & $51.10 \%$ \\
& GAR & $1.21 \%$ & $-0.64 \%$ & $0.63 \%$ & $1.47 \%$ \\
\hline \multirow{2}{*}{ LAD } & RMSE & 1.0123 & 1.0098 & 1.0156 & 1.0155 \\
& MAE & 1.0090 & 1.0062 & 1.0090 & 1.0127 \\
& PC & $51.77 \%$ & $52.27 \%$ & $52.60 \%$ & $50.63 \%$ \\
& GAR & $1.52 \%$ & $-1.17 \%$ & $4.51 \%$ & $1.47 \%$
\end{tabular}

\begin{tabular}{ll|c|c|c|c|c} 
& & EURUSD & EURCHF & EURJPY & EURGBP & Portfolio \\
\hline \multirow{2}{*}{ OLS } & RMSE & 1.0064 & 1.0112 & 1.0101 & 1.0123 & \\
& MAE & 1.0032 & 1.0067 & 1.0079 & 1.0102 & \\
& PC & $53.40 \%$ & $48.80 \%$ & $51.20 \%$ & $52.80 \%$ & $51.40 \%$ \\
& GAR & $5.01 \%$ & $-2.78 \%$ & $-0.80 \%$ & $-2.24 \%$ & $-0.13 \%$ \\
\hline \multirow{2}{*}{ MLE-t } & RMSE & 1.0069 & 1.0098 & 1.0090 & 1.0128 & \\
& MAE & 1.0041 & 1.0037 & 1.0050 & 1.0105 & \\
& PC & $53.80 \%$ & $51.30 \%$ & $50.40 \%$ & $52.60 \%$ & $51.87 \%$ \\
& GAR & $5.22 \%$ & $0.36 \%$ & $-2.36 \%$ & $-0.94 \%$ & $0.62 \%$ \\
\hline \multirow{2}{*}{ LAD } & RMSE & 1.0069 & 1.0074 & 1.0152 & 1.0195 & \\
& MAE & 1.0058 & 1.0012 & 1.0103 & 1.0190 & \\
& PC & $51.80 \%$ & $54.10 \%$ & $52.00 \%$ & $51.20 \%$ & $52.05 \%$ \\
& GAR & $0.70 \%$ & $2.54 \%$ & $-1.96 \%$ & $-0.72 \%$ & $0.86 \%$
\end{tabular}

See notes to Table 3.1.1 for details on cell entries. 
Table 3.2.2

Forecasts based on rolling window

First differenced natural log nominal exchange rate $\left(\Delta l s_{t}\right)$

Window size of $\boldsymbol{n}_{T}=\mathbf{5 0 0}$

\begin{tabular}{ll|c|c|c|c} 
& & GBPUSD & USDCHF & USDJPY & USDCAD \\
\hline \multirow{2}{*}{ OLS } & RMSE & 1.0008 & 1.0004 & 1.0031 & 0.9998 \\
& MAE & 1.0000 & 0.9975 & 0.9988 & 0.9997 \\
& PC & $52.30 \%$ & $53.43 \%$ & $52.23 \%$ & $51.03 \%$ \\
& GAR & $2.82 \%$ & $3.21 \%$ & $0.84 \%$ & $0.67 \%$ \\
\hline \multirow{2}{*}{ MLE-t } & RMSE & 1.0010 & 1.0001 & 1.0052 & 0.9998 \\
& MAE & 0.9997 & 0.9953 & 0.9984 & 1.0000 \\
& PC & $51.90 \%$ & $53.83 \%$ & $51.47 \%$ & $50.67 \%$ \\
& GAR & $2.85 \%$ & $3.81 \%$ & $-1.58 \%$ & $0.55 \%$ \\
\hline \multirow{2}{*}{ LAD } & RMSE & 1.0014 & 1.0010 & 1.0077 & 0.9998 \\
& MAE & 0.9992 & 0.9955 & 1.0018 & 1.0007 \\
& PC & $51.77 \%$ & $54.63 \%$ & $50.73 \%$ & $51.70 \%$ \\
& GAR & $2.78 \%$ & $6.05 \%$ & $-2.24 \%$ & $2.03 \%$
\end{tabular}

\begin{tabular}{ll|c|c|c|c|c} 
& & EURUSD & EURCHF & EURJPY & EURGBP & Portfolio \\
\hline \multirow{2}{*}{ OLS } & RMSE & 0.9988 & 1.0005 & 1.0024 & 1.0013 & \\
& MAE & 0.9959 & 0.9963 & 1.0002 & 1.0036 & \\
& PC & $53.10 \%$ & $51.80 \%$ & $52.10 \%$ & $50.60 \%$ & $52.07 \%$ \\
& GAR & $6.49 \%$ & $-0.11 \%$ & $1.92 \%$ & $0.74 \%$ & $2.07 \%$ \\
\hline \multirow{2}{*}{ MLE-t } & RMSE & 0.9990 & 1.0014 & 1.0024 & 1.0010 & \\
& MAE & 0.9963 & 0.9957 & 0.9990 & 1.0026 & \\
& PC & $53.30 \%$ & $53.50 \%$ & $53.20 \%$ & $51.30 \%$ & $52.40 \%$ \\
& GAR & $6.10 \%$ & $2.24 \%$ & $4.52 \%$ & $-0.10 \%$ & $2.30 \%$ \\
\hline \multirow{2}{*}{ LAD } & RMSE & 0.9998 & 1.0028 & 1.0037 & 1.0025 & \\
& MAE & 0.9968 & 0.9963 & 0.9981 & 1.0033 & \\
& PC & $52.50 \%$ & $55.30 \%$ & $54.30 \%$ & $52.00 \%$ & $52.87 \%$ \\
& GAR & $4.40 \%$ & $4.66 \%$ & $5.47 \%$ & $3.18 \%$ & $3.29 \%$
\end{tabular}

See notes to Table 3.1.1 for details on cell entries. 
Table 3.2.3

Forecasts based on rolling window

First differenced natural log nominal exchange rate $\left(\Delta l s_{t}\right)$

Window size of $n_{T}=1000$

\begin{tabular}{ll|c|c|c|c} 
& & GBPUSD & USDCHF & USDJPY & USDCAD \\
\hline \multirow{2}{*}{ OLS } & RMSE & 1.0030 & 0.9993 & 1.0020 & 0.9990 \\
& MAE & 1.0026 & 0.9981 & 1.0006 & 0.9993 \\
& PC & $50.10 \%$ & $52.37 \%$ & $48.37 \%$ & $52.27 \%$ \\
& GAR & $-2.57 \%$ & $1.28 \%$ & $-9.10 \%$ & $3.90 \%$ \\
\hline \multirow{2}{*}{ MLE-t } & RMSE & 1.0026 & 0.9993 & 1.0047 & 0.9988 \\
& MAE & 1.0021 & 0.9963 & 1.0002 & 0.9990 \\
& PC & $50.53 \%$ & $53.17 \%$ & $50.53 \%$ & $52.23 \%$ \\
& GAR & $-1.82 \%$ & $3.34 \%$ & $0.25 \%$ & $4.16 \%$ \\
\hline \multirow{2}{*}{ LAD } & RMSE & 1.0026 & 0.9997 & 1.0040 & 0.9988 \\
& MAE & 1.0018 & 0.9963 & 1.0006 & 0.9993 \\
& PC & $51.43 \%$ & $53.70 \%$ & $50.10 \%$ & $51.93 \%$ \\
& GAR & $-0.09 \%$ & $4.69 \%$ & $-0.80 \%$ & $4.36 \%$
\end{tabular}

\begin{tabular}{ll|c|c|c|c|c} 
& & EURUSD & EURCHF & EURJPY & EURGBP & Portfolio \\
\hline \multirow{2}{*}{ OLS } & RMSE & 0.9993 & 0.9993 & 1.0005 & 1.0018 & \\
& MAE & 0.9972 & 0.9972 & 0.9983 & 1.0046 & \\
& PC & $53.70 \%$ & $50.90 \%$ & $53.70 \%$ & $48.00 \%$ & $51.18 \%$ \\
& GAR & $5.92 \%$ & $-0.64 \%$ & $2.87 \%$ & $-4.72 \%$ & $-0.38 \%$ \\
\hline \multirow{2}{*}{ MLE-t } & RMSE & 1.0000 & 1.0000 & 1.0005 & 1.0020 & \\
& MAE & 0.9978 & 0.9963 & 0.9971 & 1.0049 & \\
& PC & $54.00 \%$ & $52.00 \%$ & $54.80 \%$ & $48.20 \%$ & $51.93 \%$ \\
& GAR & $7.66 \%$ & $-0.05 \%$ & $6.12 \%$ & $-3.71 \%$ & $1.99 \%$ \\
\hline \multirow{2}{*}{ LAD } & RMSE & 1.0002 & 1.0005 & 1.0015 & 1.0025 & \\
& MAE & 0.9972 & 0.9957 & 0.9957 & 1.0062 & \\
& PC & $55.40 \%$ & $53.80 \%$ & $55.10 \%$ & $49.30 \%$ & $52.60 \%$ \\
& GAR & $13.77 \%$ & $1.90 \%$ & $7.86 \%$ & $-3.14 \%$ & $3.57 \%$
\end{tabular}

See notes to Table 3.1.1 for details on cell entries. 
Table 4.1

Forecasts based on expanding window

First differenced nominal exchange rate $\left(\Delta s_{t}\right)$

\begin{tabular}{ll|c|c|c|c} 
& & GBPUSD & USDCHF & USDJPY & USDCAD \\
\hline \multirow{2}{*}{ OLS } & RMSE & 1.0004 & 0.9996 & 1.0008 & 1.0000 \\
& MAE & 1.0006 & 0.9993 & 0.9998 & 1.0002 \\
& PC & $49.33 \%$ & $51.97 \%$ & $49.27 \%$ & $51.23 \%$ \\
& GAR & $-3.74 \%$ & $5.35 \%$ & $-7.51 \%$ & $2.24 \%$ \\
\hline \multirow{2}{*}{ MLE-t } & RMSE & 1.0005 & 0.9984 & 1.0019 & 0.9998 \\
& MAE & 0.9989 & 0.9960 & 0.9985 & 1.0002 \\
& PC & $52.13 \%$ & $54.70 \%$ & $51.03 \%$ & $50.77 \%$ \\
& GAR & $1.75 \%$ & $6.98 \%$ & $0.83 \%$ & $0.61 \%$ \\
\hline \multirow{2}{*}{ LAD } & RMSE & 1.0002 & 0.9982 & 1.0024 & 0.9998 \\
& MAE & 0.9987 & 0.9953 & 0.9986 & 1.0002 \\
& PC & $51.93 \%$ & $54.47 \%$ & $52.03 \%$ & $51.07 \%$ \\
& GAR & $1.10 \%$ & $9.40 \%$ & $0.76 \%$ & $3.22 \%$
\end{tabular}

\begin{tabular}{ll|c|c|c|c|c} 
& & EURUSD & EURCHF & EURJPY & EURGBP & Portfolio \\
\hline OLS & RMSE & 0.9985 & 1.0006 & 1.0003 & 1.0029 & \\
& MAE & 0.9961 & 0.9988 & 1.0003 & 1.0043 & \\
& PC & $52.50 \%$ & $49.80 \%$ & $51.30 \%$ & $49.70 \%$ & $50.64 \%$ \\
& GAR & $6.03 \%$ & $-1.29 \%$ & $-1.95 \%$ & $-3.31 \%$ & $-0.52 \%$ \\
\hline \multirow{2}{*}{ MLE-t } & RMSE & 0.9986 & 1.0006 & 0.9999 & 1.0040 & \\
& MAE & 0.9963 & 0.9976 & 0.9988 & 1.0053 & \\
& PC & $52.90 \%$ & $50.80 \%$ & $53.60 \%$ & $49.60 \%$ & $51.94 \%$ \\
& GAR & $6.63 \%$ & $-1.24 \%$ & $4.95 \%$ & $-4.07 \%$ & $2.06 \%$ \\
\hline \multirow{2}{*}{ LAD } & RMSE & 0.9986 & 1.0006 & 0.9997 & 1.0047 & \\
& MAE & 0.9959 & 0.9968 & 0.9962 & 1.0062 & \\
& PC & $52.20 \%$ & $52.10 \%$ & $54.20 \%$ & $51.50 \%$ & $52.44 \%$ \\
& GAR & $5.80 \%$ & $0.36 \%$ & $6.12 \%$ & $-1.74 \%$ & $3.13 \%$
\end{tabular}

See notes to Table 3.1.1 for details on cell entries. 
Table 4.2

Forecasts based on expanding window

First differenced natural log nominal exchange rate $\left(\Delta l s_{\imath}\right)$

\begin{tabular}{ll|c|c|c|c} 
& & GBPUSD & USDCHF & USDJPY & USDCAD \\
\hline \multirow{2}{*}{ OLS } & RMSE & 1.0002 & 0.9999 & 1.0007 & 1.0000 \\
& MAE & 1.0000 & 1.0000 & 0.9996 & 1.0003 \\
& PC & $49.87 \%$ & $51.43 \%$ & $49.03 \%$ & $50.83 \%$ \\
& GAR & $-4.06 \%$ & $3.67 \%$ & $-8.06 \%$ & $0.98 \%$ \\
\hline \multirow{2}{*}{ MLE-t } & RMSE & 1.0006 & 0.9984 & 1.0017 & 0.9998 \\
& MAE & 0.9989 & 0.9959 & 0.9982 & 1.0003 \\
& PC & $51.83 \%$ & $54.60 \%$ & $51.13 \%$ & $50.40 \%$ \\
& GAR & $0.96 \%$ & $6.58 \%$ & $0.80 \%$ & $-0.16 \%$ \\
\hline \multirow{2}{*}{ LAD } & RMSE & 1.0006 & 0.9981 & 1.0018 & 0.9998 \\
& MAE & 0.9987 & 0.9953 & 0.9982 & 1.0003 \\
& PC & $52.40 \%$ & $54.27 \%$ & $51.93 \%$ & $50.67 \%$ \\
& GAR & $1.91 \%$ & $8.68 \%$ & $0.01 \%$ & $2.23 \%$
\end{tabular}

\begin{tabular}{ll|c|c|c|c|c} 
& & EURUSD & EURCHF & EURJPY & EURGBP & Portfolio \\
\hline \multirow{2}{*}{ OLS } & RMSE & 0.9983 & 1.0005 & 1.0002 & 1.0030 & \\
& MAE & 0.9961 & 0.9988 & 1.0005 & 1.0039 & \\
& PC & $52.80 \%$ & $49.80 \%$ & $50.10 \%$ & $49.60 \%$ & $50.43 \%$ \\
& GAR & $6.71 \%$ & $-1.29 \%$ & $-5.24 \%$ & $-3.53 \%$ & $-1.35 \%$ \\
\hline \multirow{2}{*}{ MLE-t } & RMSE & 0.9987 & 1.0009 & 0.9998 & 1.0043 & \\
& MAE & 0.9963 & 0.9975 & 0.9988 & 1.0056 & \\
& PC & $52.80 \%$ & $50.70 \%$ & $53.20 \%$ & $49.50 \%$ & $51.77 \%$ \\
& GAR & $6.57 \%$ & $-1.24 \%$ & $1.75 \%$ & $-4.05 \%$ & $1.40 \%$ \\
\hline \multirow{2}{*}{ LAD } & RMSE & 0.9985 & 1.0005 & 0.9998 & 1.0053 & \\
& MAE & 0.9959 & 0.9969 & 0.9959 & 1.0062 & \\
& PC & $52.10 \%$ & $52.10 \%$ & $54.10 \%$ & $51.50 \%$ & $52.38 \%$ \\
& GAR & $4.88 \%$ & $0.29 \%$ & $4.57 \%$ & $-1.82 \%$ & $2.59 \%$
\end{tabular}

See notes to Table 3.1.1 for details on cell entries. 
Table 5.1.1

Forecasts based on optimal samples

First differenced nominal exchange rate $\left(\Delta s_{t}\right)$

Sample optimization based on MSE

\begin{tabular}{ll|c|c|c|c} 
& & GBPUSD & USDCHF & USDJPY & USDCAD \\
\hline \multirow{2}{*}{ OLS } & RMSE & 1.0038 & 1.0038 & 1.0059 & 1.0022 \\
& MAE & 1.0013 & 1.0025 & 1.0047 & 1.0019 \\
& PC & $52.10 \%$ & $53.97 \%$ & $52.13 \%$ & $51.23 \%$ \\
& GAR & $1.92 \%$ & $7.95 \%$ & $0.07 \%$ & $1.16 \%$ \\
\hline \multirow{2}{*}{ MLE-t } & RMSE & 1.0032 & 1.0024 & 1.0079 & 1.0022 \\
& MAE & 1.0013 & 0.9992 & 1.0039 & 1.0014 \\
& PC & $51.80 \%$ & $54.00 \%$ & $52.10 \%$ & $51.33 \%$ \\
& GAR & $1.29 \%$ & $8.32 \%$ & $1.40 \%$ & $0.40 \%$ \\
\hline \multirow{2}{*}{ LAD } & RMSE & 1.0024 & 1.0037 & 1.0083 & 1.0033 \\
& MAE & 1.0014 & 0.9999 & 1.0049 & 1.0036 \\
& PC & $52.10 \%$ & $53.93 \%$ & $52.27 \%$ & $51.10 \%$ \\
& GAR & $5.21 \%$ & $8.34 \%$ & $1.74 \%$ & $2.24 \%$
\end{tabular}

\begin{tabular}{ll|c|c|c|c|c} 
& & EURUSD & EURCHF & EURJPY & EURGBP & Portfolio \\
\hline \multirow{2}{*}{ OLS } & RMSE & 1.0037 & 1.0060 & 1.0080 & 1.0084 & \\
& MAE & 1.0057 & 1.0044 & 1.0030 & 1.0115 & \\
& PC & $51.80 \%$ & $52.40 \%$ & $52.90 \%$ & $50.70 \%$ & $52.15 \%$ \\
& GAR & $6.41 \%$ & $0.54 \%$ & $1.48 \%$ & $-2.61 \%$ & $2.12 \%$ \\
\hline \multirow{2}{*}{ MLE-t } & RMSE & 1.0041 & 1.0039 & 1.0074 & 1.0084 & \\
& MAE & 1.0062 & 1.0012 & 1.0014 & 1.0120 & \\
& PC & $51.60 \%$ & $53.40 \%$ & $53.60 \%$ & $50.90 \%$ & $52.34 \%$ \\
& GAR & $5.03 \%$ & $2.05 \%$ & $3.58 \%$ & $-1.37 \%$ & $2.59 \%$ \\
\hline \multirow{2}{*}{ LAD } & RMSE & 1.0048 & 1.0057 & 1.0127 & 1.0150 & \\
& MAE & 1.0078 & 1.0060 & 1.0089 & 1.0201 & \\
& PC & $51.00 \%$ & $53.10 \%$ & $52.70 \%$ & $50.70 \%$ & $52.11 \%$ \\
& GAR & $4.29 \%$ & $2.56 \%$ & $3.08 \%$ & $-1.73 \%$ & $3.22 \%$
\end{tabular}

See notes to Table 3.1.1 for details on cell entries. 
Table 5.1.2

Forecasts based on optimal samples

First differenced nominal exchange rate $\left(\Delta s_{t}\right)$

Sample optimization based on MAE

\begin{tabular}{ll|c|c|c|c} 
& & GBPUSD & USDCHF & USDJPY & USDCAD \\
\hline \multirow{2}{*}{ OLS } & RMSE & 1.0042 & 1.0042 & 1.0049 & 1.0013 \\
& MAE & 1.0022 & 1.0047 & 1.0041 & 1.0007 \\
& PC & $52.17 \%$ & $53.10 \%$ & $51.37 \%$ & $51.17 \%$ \\
& GAR & $2.25 \%$ & $6.52 \%$ & $-0.35 \%$ & $1.49 \%$ \\
\hline \multirow{2}{*}{ MLE-t } & RMSE & 1.0034 & 1.0025 & 1.0071 & 1.0013 \\
& MAE & 1.0022 & 1.0010 & 1.0034 & 1.0005 \\
& PC & $52.17 \%$ & $53.43 \%$ & $52.40 \%$ & $50.97 \%$ \\
& GAR & $3.18 \%$ & $8.65 \%$ & $3.91 \%$ & $-0.10 \%$ \\
\hline \multirow{2}{*}{ LAD } & RMSE & 1.0035 & 1.0035 & 1.0062 & 1.0016 \\
& MAE & 1.0021 & 1.0024 & 1.0033 & 1.0014 \\
& PC & $52.80 \%$ & $53.40 \%$ & $52.43 \%$ & $51.33 \%$ \\
& GAR & $6.49 \%$ & $8.71 \%$ & $4.26 \%$ & $2.55 \%$
\end{tabular}

\begin{tabular}{ll|c|c|c|c|c} 
& & EURUSD & EURCHF & EURJPY & EURGBP & Portfolio \\
\hline \multirow{2}{*}{ OLS } & RMSE & 1.0027 & 1.0069 & 1.0097 & 1.0084 & \\
& MAE & 1.0030 & 1.0044 & 1.0043 & 1.0105 & \\
& PC & $52.20 \%$ & $52.60 \%$ & $52.60 \%$ & $51.80 \%$ & $52.13 \%$ \\
& GAR & $7.71 \%$ & $0.96 \%$ & $1.18 \%$ & $-0.09 \%$ & $2.46 \%$ \\
\hline \multirow{2}{*}{ MLE-t } & RMSE & 1.0027 & 1.0051 & 1.0091 & 1.0088 & \\
& MAE & 1.0034 & 1.0020 & 1.0028 & 1.0105 & \\
& PC & $52.10 \%$ & $53.30 \%$ & $53.70 \%$ & $51.50 \%$ & $52.45 \%$ \\
& GAR & $8.29 \%$ & $1.32 \%$ & $4.39 \%$ & $0.17 \%$ & $3.73 \%$ \\
\hline \multirow{2}{*}{ LAD } & RMSE & 1.0040 & 1.0075 & 1.0154 & 1.0121 & \\
& MAE & 1.0059 & 1.0068 & 1.0109 & 1.0148 & \\
& PC & $52.20 \%$ & $53.30 \%$ & $53.10 \%$ & $51.00 \%$ & $52.45 \%$ \\
& GAR & $7.54 \%$ & $2.12 \%$ & $3.19 \%$ & $-1.62 \%$ & $4.16 \%$
\end{tabular}

See notes to Table 3.1.1 for details on cell entries. 
Table 5.2.1

Forecasts based on optimal samples

First differenced natural log nominal exchange rate $\left(\Delta l_{t}\right)$

Sample optimization based on MSE

\begin{tabular}{ll|c|c|c|c} 
& & GBPUSD & USDCHF & USDJPY & USDCAD \\
\hline \multirow{2}{*}{ OLS } & RMSE & 1.0052 & 1.0057 & 1.0062 & 1.0010 \\
& MAE & 1.0011 & 1.0062 & 1.0055 & 1.0017 \\
& PC & $52.60 \%$ & $53.00 \%$ & $51.33 \%$ & $50.47 \%$ \\
& GAR & $1.73 \%$ & $8.06 \%$ & $-0.68 \%$ & $0.09 \%$ \\
\hline \multirow{2}{*}{ MLE-t } & RMSE & 1.0050 & 1.0044 & 1.0075 & 1.0010 \\
& MAE & 1.0016 & 1.0037 & 1.0045 & 1.0020 \\
& PC & $52.23 \%$ & $52.90 \%$ & $52.07 \%$ & $50.00 \%$ \\
& GAR & $0.59 \%$ & $6.69 \%$ & $3.77 \%$ & $-1.52 \%$ \\
\hline \multirow{2}{*}{ LAD } & RMSE & 1.0052 & 1.0055 & 1.0081 & 1.0017 \\
& MAE & 1.0024 & 1.0043 & 1.0045 & 1.0033 \\
& PC & $52.73 \%$ & $52.93 \%$ & $52.47 \%$ & $50.30 \%$ \\
& GAR & $5.75 \%$ & $6.83 \%$ & $4.27 \%$ & $0.94 \%$
\end{tabular}

\begin{tabular}{ll|c|c|c|c|c} 
& & EURUSD & EURCHF & EURJPY & EURGBP & Portfolio \\
\hline \multirow{2}{*}{ OLS } & RMSE & 1.0054 & 1.0065 & 1.0090 & 1.0085 & \\
& MAE & 1.0073 & 1.0055 & 1.0031 & 1.0121 & \\
& PC & $52.70 \%$ & $52.00 \%$ & $52.50 \%$ & $50.50 \%$ & $51.89 \%$ \\
& GAR & $8.14 \%$ & $0.04 \%$ & $0.29 \%$ & $-2.17 \%$ & $1.94 \%$ \\
\hline \multirow{2}{*}{ MLE-t } & RMSE & 1.0053 & 1.0042 & 1.0077 & 1.0085 & \\
& MAE & 1.0078 & 1.0025 & 1.0021 & 1.0121 & \\
& PC & $52.70 \%$ & $53.00 \%$ & $53.30 \%$ & $50.70 \%$ & $52.11 \%$ \\
& GAR & $8.58 \%$ & $1.77 \%$ & $3.27 \%$ & $-0.81 \%$ & $2.79 \%$ \\
\hline \multirow{2}{*}{ LAD } & RMSE & 1.0054 & 1.0065 & 1.0143 & 1.0155 & \\
& MAE & 1.0095 & 1.0067 & 1.0105 & 1.0203 & \\
& PC & $51.80 \%$ & $52.80 \%$ & $52.40 \%$ & $50.90 \%$ & $52.04 \%$ \\
& GAR & $7.38 \%$ & $2.08 \%$ & $2.18 \%$ & $-1.61 \%$ & $3.48 \%$
\end{tabular}

See notes to Table 3.1.1 for details on cell entries. 
Table 5.2.2

Forecasts based on optimal samples

First differenced natural log nominal exchange rate $\left(\Delta l s_{t}\right)$

Sample optimization based on MAE

\begin{tabular}{ll|c|c|c|c} 
& & GBPUSD & USDCHF & USDJPY & USDCAD \\
\hline \multirow{2}{*}{ OLS } & RMSE & 1.0048 & 1.0050 & 1.0062 & 1.0007 \\
& MAE & 1.0013 & 1.0049 & 1.0049 & 1.0017 \\
& PC & $52.97 \%$ & $52.57 \%$ & $51.23 \%$ & $50.13 \%$ \\
& GAR & $2.29 \%$ & $6.41 \%$ & $-2.38 \%$ & $0.13 \%$ \\
\hline \multirow{2}{*}{ MLE-t } & RMSE & 1.0038 & 1.0032 & 1.0075 & 1.0007 \\
& MAE & 1.0008 & 1.0016 & 1.0037 & 1.0020 \\
& PC & $53.40 \%$ & $53.07 \%$ & $52.10 \%$ & $50.40 \%$ \\
& GAR & $4.95 \%$ & $7.10 \%$ & $2.75 \%$ & $-0.62 \%$ \\
\hline \multirow{2}{*}{ LAD } & RMSE & 1.0028 & 1.0039 & 1.0065 & 1.0012 \\
& MAE & 0.9992 & 1.0019 & 1.0031 & 1.0030 \\
& PC & $53.57 \%$ & $53.33 \%$ & $52.67 \%$ & $50.50 \%$ \\
& GAR & $7.18 \%$ & $7.60 \%$ & $4.47 \%$ & $1.06 \%$
\end{tabular}

\begin{tabular}{ll|c|c|c|c|c} 
& & EURUSD & EURCHF & EURJPY & EURGBP & Portfolio \\
\hline \multirow{2}{*}{ OLS } & RMSE & 1.0061 & 1.0070 & 1.0113 & 1.0095 & \\
& MAE & 1.0060 & 1.0049 & 1.0050 & 1.0108 & \\
& PC & $51.90 \%$ & $52.40 \%$ & $52.40 \%$ & $52.30 \%$ & $51.99 \%$ \\
& GAR & $4.12 \%$ & $0.76 \%$ & $-0.53 \%$ & $-0.90 \%$ & $1.24 \%$ \\
\hline \multirow{2}{*}{ MLE-t } & RMSE & 1.0063 & 1.0051 & 1.0099 & 1.0100 & \\
& MAE & 1.0067 & 1.0018 & 1.0041 & 1.0111 & \\
& PC & $52.00 \%$ & $53.40 \%$ & $53.70 \%$ & $52.00 \%$ & $52.51 \%$ \\
& GAR & $4.98 \%$ & $1.65 \%$ & $4.21 \%$ & $-0.12 \%$ & $3.11 \%$ \\
\hline \multirow{2}{*}{ LAD } & RMSE & 1.0053 & 1.0070 & 1.0167 & 1.0158 & \\
& MAE & 1.0067 & 1.0061 & 1.0105 & 1.0177 & \\
& PC & $51.70 \%$ & $53.50 \%$ & $52.90 \%$ & $51.10 \%$ & $52.41 \%$ \\
& GAR & $3.95 \%$ & $2.11 \%$ & $2.78 \%$ & $-1.64 \%$ & $3.44 \%$
\end{tabular}

See notes to Table 3.1.1 for details on cell entries. 
Table 6.1

Forecasts based on LAD-BI estimates

First differenced nominal exchange rate $\left(\Delta s_{t}\right)$

Sample optimization based on MSE

\begin{tabular}{ll|c|c|c|c} 
& & GBPUSD & USDCHF & USDJPY & USDCAD \\
\hline LAD-BI & RMSE & 1.0026 & 1.0045 & 1.0086 & 1.0033 \\
FTC & MAE & 1.0019 & 1.0012 & 1.0056 & 1.0036 \\
& PC & $52.27 \%$ & $53.77 \%$ & $52.70 \%$ & $50.60 \%$ \\
& GAR & $5.38 \%$ & $7.87 \%$ & $3.72 \%$ & $-0.83 \%$ \\
\hline LAD-BI & RMSE & 1.0029 & 1.0056 & 1.0087 & 1.0034 \\
KTC & MAE & 1.0021 & 1.0017 & 1.0060 & 1.0039 \\
& PC & $52.40 \%$ & $54.03 \%$ & $52.77 \%$ & $50.50 \%$ \\
& GAR & $5.78 \%$ & $9.75 \%$ & $3.92 \%$ & $-1.11 \%$
\end{tabular}

\begin{tabular}{ll|c|c|c|c|c} 
& & EURUSD & EURCHF & EURJPY & EURGBP & Portfolio \\
\hline LAD-BI & RMSE & 1.0034 & 1.0081 & 1.0120 & 1.0135 & \\
FTC & MAE & 1.0071 & 1.0068 & 1.0085 & 1.0201 & \\
& PC & $51.20 \%$ & $53.10 \%$ & $53.30 \%$ & $50.00 \%$ & $52.12 \%$ \\
& GAR & $3.67 \%$ & $2.75 \%$ & $5.55 \%$ & $-2.60 \%$ & $3.19 \%$ \\
\hline LAD-BI & RMSE & 1.0038 & 1.0088 & 1.0123 & 1.0121 & \\
KTC & MAE & 1.0084 & 1.0076 & 1.0085 & 1.0196 & \\
& PC & $51.10 \%$ & $53.20 \%$ & $53.50 \%$ & $50.20 \%$ & $52.21 \%$ \\
& GAR & $4.31 \%$ & $3.13 \%$ & $5.52 \%$ & $-2.45 \%$ & $3.61 \%$
\end{tabular}

LAD-BI FTC denotes LAD-BI estimates based on a fixed tuning constant, and LAD-BI KTC denotes LAD-BI estimates based on a tuning constant adjusted for estimation sample kurtosis. See section 4 for further details. See notes to Table 3.1.1 for further details on cell entries. 
Table 6.2

Forecasts based on LAD-BI estimates

First differenced nominal exchange rate $\left(\Delta s_{t}\right)$

Sample optimization based on MAE

\begin{tabular}{ll|c|c|c|c} 
& & GBPUSD & USDCHF & USDJPY & USDCAD \\
\hline LAD-BI & RMSE & 1.0026 & 1.0033 & 1.0065 & 1.0020 \\
FTC & MAE & 1.0013 & 1.0021 & 1.0043 & 1.0019 \\
& PC & $52.90 \%$ & $53.60 \%$ & $52.67 \%$ & $50.20 \%$ \\
& GAR & $7.47 \%$ & $7.53 \%$ & $5.57 \%$ & $-1.31 \%$ \\
\hline LAD-BI & RMSE & 1.0028 & 1.0041 & 1.0061 & 1.0024 \\
KTC & MAE & 1.0014 & 1.0026 & 1.0037 & 1.0024 \\
& PC & $52.50 \%$ & $53.73 \%$ & $52.97 \%$ & $50.37 \%$ \\
& GAR & $7.36 \%$ & $9.61 \%$ & $6.14 \%$ & $-1.04 \%$
\end{tabular}

\begin{tabular}{ll|c|c|c|c|c} 
& & EURUSD & EURCHF & EURJPY & EURGBP & Portfolio \\
\hline LAD-BI & RMSE & 1.0023 & 1.0097 & 1.0141 & 1.0124 & \\
FTC & MAE & 1.0050 & 1.0072 & 1.0103 & 1.0177 & \\
& PC & $52.70 \%$ & $54.00 \%$ & $53.60 \%$ & $50.70 \%$ & $52.55 \%$ \\
& GAR & $10.71 \%$ & $3.11 \%$ & $6.34 \%$ & $-2.07 \%$ & $4.67 \%$ \\
\hline LAD-BI & RMSE & 1.0022 & 1.0094 & 1.0146 & 1.0117 & \\
KTC & MAE & 1.0050 & 1.0076 & 1.0108 & 1.0168 & \\
& PC & $52.80 \%$ & $54.30 \%$ & $53.70 \%$ & $51.30 \%$ & $52.71 \%$ \\
& GAR & $10.40 \%$ & $3.49 \%$ & $6.52 \%$ & $-0.97 \%$ & $5.19 \%$
\end{tabular}

See notes to Table 6.1 for details on cell entries. 
Table 7.1

Statistical significance

LAD estimates, with sample optimization based on MAE

\begin{tabular}{cc|c|c|c|c} 
& & GBPUSD & USDCHF & USDJPY & USDCAD \\
\hline \multirow{2}{*}{ LAD } & PT & 3.0427 & 3.4485 & 2.4437 & 1.7309 \\
& & {$[0.001]$} & {$[0.000]$} & {$[0.007]$} & {$[0.042]$} \\
& DM & 2.6021 & 3.4310 & 2.5539 & 2.4277 \\
& & {$[0.007]$} & {$[0.002]$} & {$[0.012]$} & {$[0.023]$} \\
& \multirow{4}{*}{ GAR } & $6.49 \%$ & $8.71 \%$ & $4.26 \%$ & $2.55 \%$ \\
& & {$[0.002]$} & {$[0.016]$} & {$[0.031]$} & {$[0.155]$}
\end{tabular}

\begin{tabular}{cc|c|c|c|c} 
& & EURUSD & EURCHF & EURJPY & EURGBP \\
\hline \multirow{2}{*}{ LAD } & PT & 1.2881 & 1.1261 & -1.2151 & 0.6789 \\
& & {$[0.099]$} & {$[0.130]$} & {$[0.888]$} & {$[0.249]$} \\
& DM & 2.5545 & 1.9850 & 1.0735 & 0.0378 \\
& & {$[0.008]$} & {$[0.073]$} & {$[0.221]$} & {$[0.481]$} \\
& GAR & $7.54 \%$ & $2.12 \%$ & $3.19 \%$ & $-1.62 \%$ \\
& & {$[0.112]$} & {$[0.086]$} & {$[0.446]$} & {$[0.738]$}
\end{tabular}

Each panel contains test statistics and p-values in brackets. PT denotes the PesaranTimmermann (1992) test for market timing ability. DM denotes the modified DieboldMariano (1995) test. GAR is the gross annualized return. P-values for the DM test and GAR are based on a semi-parametric bootstrap. See section 8 for complete details. 
Table 7.2

Statistical significance

LAD-BI estimates, with sample optimization based on MAE

\begin{tabular}{ll|c|c|c|c} 
& & GBPUSD & USDCHF & USDJPY & USDCAD \\
\hline LAD-BI & PT & 3.1480 & 3.6770 & 2.6721 & 0.3862 \\
FTC & & {$[0.001]$} & {$[0.000]$} & {$[0.004]$} & {$[0.350]$} \\
& DM & 2.8638 & 3.2793 & 2.7655 & 0.5232 \\
& & {$[0.003]$} & {$[0.002]$} & {$[0.007]$} & {$[0.409]$} \\
& GAR & $7.47 \%$ & $7.53 \%$ & $5.57 \%$ & $-1.31 \%$ \\
& & {$[0.000]$} & {$[0.029]$} & {$[0.012]$} & {$[0.815]$} \\
\hline \multirow{2}{*}{ LAD-BI } & PT & 2.7058 & 3.8162 & 3.0008 & 0.5779 \\
KTC & & {$[0.003]$} & {$[0.000]$} & {$[0.001]$} & {$[0.282]$} \\
& \multirow{2}{*}{ DM } & 2.8131 & 3.7466 & 2.8768 & 0.6568 \\
& & {$[0.003]$} & {$[0.000]$} & {$[0.006]$} & {$[0.370]$} \\
& \multirow{2}{*}{ GAR } & $7.36 \%$ & $9.61 \%$ & $6.14 \%$ & $-1.04 \%$ \\
& & {$[0.000]$} & {$[0.007]$} & {$[0.007]$} & {$[0.788]$}
\end{tabular}

\begin{tabular}{ll|c|c|c|c} 
& & EURUSD & EURCHF & EURJPY & EURGBP \\
\hline LAD-BI & PT & 1.6129 & 1.6019 & -0.7352 & 0.4689 \\
FTC & & {$[0.053]$} & {$[0.055]$} & {$[0.769]$} & {$[0.320]$} \\
& \multirow{2}{*}{ DM } & 2.9892 & 2.2072 & 1.7085 & -0.0587 \\
& & {$[0.000]$} & {$[0.051]$} & {$[0.071]$} & {$[0.528]$} \\
& \multirow{2}{*}{ GAR } & $10.71 \%$ & $3.11 \%$ & $6.34 \%$ & $-2.07 \%$ \\
& & {$[0.026]$} & {$[0.036]$} & {$[0.168]$} & {$[0.801]$} \\
\hline \multirow{2}{*}{ LAD-BI } & PT & 1.6783 & 1.8225 & -0.6322 & 0.8513 \\
KTC & & {$[0.047]$} & {$[0.034]$} & {$[0.736]$} & {$[0.197]$} \\
& \multirow{2}{*}{ DM } & 2.9425 & 2.3315 & 1.7390 & 0.1788 \\
& & {$[0.000]$} & {$[0.041]$} & {$[0.064]$} & {$[0.394]$} \\
& \multirow{2}{*}{ GAR } & $10.40 \%$ & $3.49 \%$ & $6.52 \%$ & $-0.97 \%$ \\
& & {$[0.029]$} & {$[0.020]$} & {$[0.155]$} & {$[0.650]$}
\end{tabular}

See notes to Table 7.1 for details on cell entries. 
Table 8.1

Profitability and risk

LAD-BI KTC estimates, with sample optimization based on MAE

No transactions costs

\begin{tabular}{l|c|c|c|c} 
& GBPUSD & USDCHF & USDJPY & USDCAD \\
\hline NAR & $7.36 \%$ & $9.61 \%$ & $6.14 \%$ & $-1.04 \%$ \\
AV & $8.07 \%$ & $11.00 \%$ & $11.32 \%$ & $6.44 \%$ \\
SR & 0.9122 & 0.8733 & 0.5423 & -0.1614 \\
IR & 0.0758 & 0.0738 & 0.0502 & -0.0108 \\
NT & 994 & 974 & 966 & 1158 \\
PG & 0.5217 & 0.5327 & 0.5283 & 0.5003 \\
PL & 0.4783 & 0.4673 & 0.4717 & 0.4997 \\
MG & $0.39 \%$ & $0.52 \%$ & $0.51 \%$ & $0.30 \%$ \\
ML & $-0.37 \%$ & $-0.51 \%$ & $-0.51 \%$ & $-0.30 \%$ \\
PTL & 0.0184 & 0.0561 & 0.1392 & 1.0000
\end{tabular}

\begin{tabular}{l|c|c|c|c} 
& EURUSD & EURCHF & EURJPY & EURGBP \\
\hline NAR & $10.40 \%$ & $3.49 \%$ & $6.52 \%$ & $-0.97 \%$ \\
AV & $9.71 \%$ & $3.45 \%$ & $8.77 \%$ & $6.42 \%$ \\
SR & 1.0713 & 1.0113 & 0.7427 & -0.1516 \\
IR & 0.0867 & 0.0828 & 0.0618 & -0.0098 \\
NT & 334 & 287 & 125 & 160 \\
PG & 0.5240 & 0.5400 & 0.5460 & 0.5030 \\
PL & 0.4760 & 0.4600 & 0.4540 & 0.4970 \\
MG & $0.48 \%$ & $0.16 \%$ & $0.41 \%$ & $0.30 \%$ \\
ML & $-0.44 \%$ & $-0.16 \%$ & $-0.43 \%$ & $-0.31 \%$ \\
PTL & 0.0236 & 0.0000 & 0.0525 & 1.0000
\end{tabular}

NAR is the net annualized return, AV is the annualized volatility, SR is the Sharpe Ratio, IR is the ideal profit ratio, NT is the number of trades, PG is the sample probability of a gain on a given day, PL is the sample probability of a loss on a given day, MG is the mean gain, ML is the mean loss, and PTL is the probability of a $10 \%$ loss. 
Table 8.2

Profitability and risk

LAD-BI KTC estimates, with sample optimization based on MAE

0.5 bp one-way transactions cost

\begin{tabular}{l|c|c|c|c} 
& GBPUSD & USDCHF & USDJPY & USDCAD \\
\hline NAR & $6.45 \%$ & $8.69 \%$ & $5.26 \%$ & $-2.02 \%$ \\
AV & $8.07 \%$ & $11.00 \%$ & $11.32 \%$ & $6.44 \%$ \\
SR & 0.7990 & 0.7902 & 0.4647 & -0.3136 \\
IR & 0.0689 & 0.0689 & 0.0448 & -0.0245 \\
NT & 994 & 974 & 966 & 1158 \\
PG & 0.5177 & 0.5317 & 0.5257 & 0.4963 \\
PL & 0.4823 & 0.4683 & 0.4743 & 0.5037 \\
MG & $0.39 \%$ & $0.52 \%$ & $0.51 \%$ & $0.30 \%$ \\
ML & $-0.37 \%$ & $-0.51 \%$ & $-0.51 \%$ & $-0.31 \%$ \\
PTL & 0.0291 & 0.0718 & 0.1785 & 1.0000
\end{tabular}

\begin{tabular}{l|c|c|c|c} 
& EURUSD & EURCHF & EURJPY & EURGBP \\
\hline NAR & $9.45 \%$ & $2.73 \%$ & $6.17 \%$ & $-1.38 \%$ \\
AV & $9.71 \%$ & $3.45 \%$ & $8.77 \%$ & $6.41 \%$ \\
SR & 0.9736 & 0.7905 & 0.7037 & -0.2152 \\
IR & 0.0813 & 0.0695 & 0.0603 & -0.0155 \\
NT & 334 & 287 & 125 & 160 \\
PG & 0.5210 & 0.5380 & 0.5450 & 0.4980 \\
PL & 0.4790 & 0.4620 & 0.4550 & 0.5020 \\
MG & $0.48 \%$ & $0.16 \%$ & $0.41 \%$ & $0.30 \%$ \\
ML & $-0.45 \%$ & $-0.16 \%$ & $-0.43 \%$ & $-0.31 \%$ \\
PTL & 0.0322 & 0.0003 & 0.0605 & 1.0000
\end{tabular}

See notes to Table 8.1 for details on cell entries. 
Table 8.3

Profitability and risk

LAD-BI KTC estimates, with sample optimization based on MAE

1.0 bp one-way transactions cost

\begin{tabular}{l|c|c|c|c} 
& GBPUSD & USDCHF & USDJPY & USDCAD \\
\hline NAR & $5.54 \%$ & $7.79 \%$ & $4.39 \%$ & $-2.99 \%$ \\
AV & $8.07 \%$ & $11.00 \%$ & $11.32 \%$ & $6.45 \%$ \\
SR & 0.6867 & 0.7078 & 0.3877 & -0.4641 \\
IR & 0.0616 & 0.0637 & 0.0391 & -0.0391 \\
NT & 994 & 974 & 966 & 1158 \\
PG & 0.5140 & 0.5290 & 0.5233 & 0.4937 \\
PL & 0.4860 & 0.4710 & 0.4767 & 0.5063 \\
MG & $0.39 \%$ & $0.51 \%$ & $0.51 \%$ & $0.29 \%$ \\
ML & $-0.37 \%$ & $-0.51 \%$ & $-0.51 \%$ & $-0.31 \%$ \\
PTL & 0.0461 & 0.0918 & 0.2289 & 1.0000
\end{tabular}

\begin{tabular}{l|c|c|c|c} 
& EURUSD & EURCHF & EURJPY & EURGBP \\
\hline NAR & $8.52 \%$ & $1.97 \%$ & $5.83 \%$ & $-1.79 \%$ \\
AV & $9.71 \%$ & $3.45 \%$ & $8.77 \%$ & $6.41 \%$ \\
SR & 0.8768 & 0.5708 & 0.6647 & -0.2786 \\
IR & 0.0756 & 0.0543 & 0.0587 & -0.0217 \\
NT & 334 & 287 & 125 & 160 \\
PG & 0.5200 & 0.5340 & 0.5450 & 0.4980 \\
PL & 0.4800 & 0.4660 & 0.4550 & 0.5020 \\
MG & $0.48 \%$ & $0.16 \%$ & $0.41 \%$ & $0.30 \%$ \\
ML & $-0.45 \%$ & $-0.17 \%$ & $-0.44 \%$ & $-0.31 \%$ \\
PTL & 0.0439 & 0.0028 & 0.0698 & 1.0000
\end{tabular}

See notes to Table 8.1 for details on cell entries. 
Table 8.4

Profitability and risk

LAD-BI KTC estimates, with sample optimization based on MAE

2.0 bp one-way transactions cost

\begin{tabular}{l|c|c|c|c} 
& GBPUSD & USDCHF & USDJPY & USDCAD \\
\hline NAR & $3.75 \%$ & $6.00 \%$ & $2.67 \%$ & $-4.91 \%$ \\
AV & $8.07 \%$ & $11.01 \%$ & $11.33 \%$ & $6.46 \%$ \\
SR & 0.4648 & 0.5449 & 0.2356 & -0.7599 \\
IR & 0.0457 & 0.0526 & 0.0271 & -0.0718 \\
NT & 994 & 974 & 966 & 1158 \\
PG & 0.5100 & 0.5237 & 0.5183 & 0.4840 \\
PL & 0.4900 & 0.4763 & 0.4817 & 0.5160 \\
MG & $0.39 \%$ & $0.51 \%$ & $0.50 \%$ & $0.29 \%$ \\
ML & $-0.37 \%$ & $-0.51 \%$ & $-0.52 \%$ & $-0.31 \%$ \\
PTL & 0.1157 & 0.1505 & 0.3764 & 1.0000
\end{tabular}

\begin{tabular}{l|c|c|c|c} 
& EURUSD & EURCHF & EURJPY & EURGBP \\
\hline NAR & $6.66 \%$ & $0.47 \%$ & $5.15 \%$ & $-2.59 \%$ \\
AV & $9.72 \%$ & $3.46 \%$ & $8.77 \%$ & $6.41 \%$ \\
SR & 0.6855 & 0.1361 & 0.5870 & -0.4045 \\
IR & 0.0634 & 0.0166 & 0.0552 & -0.0353 \\
NT & 334 & 287 & 125 & 160 \\
PG & 0.5150 & 0.5250 & 0.5450 & 0.4930 \\
PL & 0.4850 & 0.4750 & 0.4550 & 0.5070 \\
MG & $0.48 \%$ & $0.16 \%$ & $0.40 \%$ & $0.30 \%$ \\
ML & $-0.45 \%$ & $-0.17 \%$ & $-0.44 \%$ & $-0.31 \%$ \\
PTL & 0.0818 & 0.2134 & 0.0927 & 1.0000
\end{tabular}

See notes to Table 8.1 for details on cell entries. 
Table 9.1

Combination of forecasts across estimators

LAD-BI + MLE-t, sample optimization based on MAE

\begin{tabular}{ll|c|c|c|c} 
Weights & & GBPUSD & USDCHF & USDJPY & USDCAD \\
\hline \multirow{2}{*}{ Equal } & RMSE & 1.0025 & 1.0029 & 1.0060 & 1.0016 \\
& MAE & 1.0013 & 1.0012 & 1.0024 & 1.0012 \\
& PC & $51.50 \%$ & $53.70 \%$ & $52.33 \%$ & $50.07 \%$ \\
& GAR & $3.42 \%$ & $9.25 \%$ & $3.39 \%$ & $-0.91 \%$ \\
\hline \multirow{2}{*}{ MAE } & RMSE & 1.0025 & 1.0028 & 1.0060 & 1.0016 \\
& MAE & 1.0013 & 1.0012 & 1.0024 & 1.0012 \\
& PC & $51.50 \%$ & $53.73 \%$ & $52.17 \%$ & $50.13 \%$ \\
& GAR & $3.39 \%$ & $9.27 \%$ & $3.15 \%$ & $-0.67 \%$ \\
\hline \multirow{2}{*}{ MSE } & RMSE & 1.0026 & 1.0027 & 1.0061 & 1.0016 \\
& MAE & 1.0014 & 1.0011 & 1.0024 & 1.0012 \\
& PC & $51.47 \%$ & $53.77 \%$ & $52.13 \%$ & $50.13 \%$ \\
& GAR & $3.23 \%$ & $9.14 \%$ & $2.97 \%$ & $-0.35 \%$
\end{tabular}

\begin{tabular}{ll|c|c|c|c|c} 
Weights & & EURUSD & EURCHF & EURJPY & EURGBP & Portfolio \\
\hline Equal & RMSE & 1.0018 & 1.0063 & 1.0108 & 1.0088 & \\
& MAE & 1.0029 & 1.0036 & 1.0057 & 1.0115 & \\
& PC & $53.60 \%$ & $53.40 \%$ & $52.90 \%$ & $50.90 \%$ & $52.30 \%$ \\
& GAR & $11.08 \%$ & $2.21 \%$ & $3.99 \%$ & $-0.74 \%$ & $3.96 \%$ \\
\hline MAE & RMSE & 1.0018 & 1.0063 & 1.0107 & 1.0088 & \\
& MAE & 1.0029 & 1.0036 & 1.0056 & 1.0115 & \\
& PC & $53.60 \%$ & $53.10 \%$ & $52.70 \%$ & $50.90 \%$ & $52.23 \%$ \\
& GAR & $11.08 \%$ & $1.87 \%$ & $3.18 \%$ & $-0.85 \%$ & $3.80 \%$ \\
\hline \multirow{2}{*}{ MSE } & RMSE & 1.0019 & 1.0063 & 1.0107 & 1.0088 & \\
& MAE & 1.0030 & 1.0040 & 1.0055 & 1.0110 & \\
& PC & $53.50 \%$ & $52.90 \%$ & $52.90 \%$ & $51.00 \%$ & $52.23 \%$ \\
& GAR & $11.17 \%$ & $1.86 \%$ & $3.46 \%$ & $-0.38 \%$ & $3.89 \%$
\end{tabular}

The first column lists the source of the weights for the linear forecast combination. See section 10 for details. LAD-BI forecasts are from the KTC specification. See notes to Table 3.1.1 for further details on cell entries. 
Table 9.2

Combination of forecasts across estimators

LAD-BI + MLE-t + OLS, sample optimization based on MAE

\begin{tabular}{ll|c|c|c|c} 
Weights & & GBPUSD & USDCHF & USDJPY & USDCAD \\
\hline \multirow{2}{*}{ Equal } & RMSE & 1.0026 & 1.0028 & 1.0050 & 1.0015 \\
& MAE & 1.0013 & 1.0018 & 1.0023 & 1.0010 \\
& PC & $52.17 \%$ & $53.40 \%$ & $52.40 \%$ & $50.47 \%$ \\
& GAR & $3.53 \%$ & $8.38 \%$ & $4.55 \%$ & $0.51 \%$ \\
\hline \multirow{2}{*}{ MAE } & RMSE & 1.0028 & 1.0028 & 1.0051 & 1.0015 \\
& MAE & 1.0013 & 1.0018 & 1.0023 & 1.0010 \\
& PC & $52.30 \%$ & $53.37 \%$ & $52.27 \%$ & $50.60 \%$ \\
& GAR & $4.19 \%$ & $8.38 \%$ & $4.52 \%$ & $0.68 \%$ \\
\hline \multirow{2}{*}{ MSE } & RMSE & 1.0029 & 1.0027 & 1.0051 & 1.0015 \\
& MAE & 1.0014 & 1.0019 & 1.0024 & 1.0010 \\
& PC & $52.60 \%$ & $53.27 \%$ & $52.37 \%$ & $50.57 \%$ \\
& GAR & $4.92 \%$ & $7.63 \%$ & $4.54 \%$ & $0.09 \%$
\end{tabular}

\begin{tabular}{ll|c|c|c|c|c} 
Weights & & EURUSD & EURCHF & EURJPY & EURGBP & Portfolio \\
\hline Equal & RMSE & 1.0018 & 1.0060 & 1.0098 & 1.0084 & \\
& MAE & 1.0025 & 1.0036 & 1.0047 & 1.0105 & \\
& PC & $53.00 \%$ & $52.80 \%$ & $53.70 \%$ & $51.40 \%$ & $52.42 \%$ \\
& GAR & $10.27 \%$ & $1.27 \%$ & $4.80 \%$ & $-0.24 \%$ & $4.13 \%$ \\
\hline MAE & RMSE & 1.0018 & 1.0060 & 1.0098 & 1.0084 & \\
& MAE & 1.0025 & 1.0036 & 1.0046 & 1.0105 & \\
& PC & $53.00 \%$ & $52.70 \%$ & $53.30 \%$ & $52.00 \%$ & $52.44 \%$ \\
& GAR & $10.45 \%$ & $1.13 \%$ & $3.81 \%$ & $0.20 \%$ & $4.17 \%$ \\
\hline \multirow{2}{*}{ MSE } & RMSE & 1.0019 & 1.0060 & 1.0098 & 1.0084 & \\
& MAE & 1.0025 & 1.0036 & 1.0046 & 1.0105 & \\
& PC & $53.00 \%$ & $52.80 \%$ & $53.40 \%$ & $51.60 \%$ & $52.45 \%$ \\
& GAR & $10.26 \%$ & $1.39 \%$ & $3.69 \%$ & $-0.19 \%$ & $4.04 \%$
\end{tabular}

See notes to Table 9.1 for details on cell entries. 
Table 10.1

Combination of forecasts across estimation samples, RW500 + RW1000

\begin{tabular}{ll|c|c|c|c} 
Weights & & GBPUSD & USDCHF & USDJPY & USDCAD \\
\hline Equal & RMSE & 1.0022 & 1.0005 & 1.0054 & 0.9998 \\
& MAE & 1.0005 & 0.9957 & 1.0012 & 1.0000 \\
& PC & $51.83 \%$ & $54.30 \%$ & $50.43 \%$ & $52.17 \%$ \\
& GAR & $1.99 \%$ & $5.77 \%$ & $-3.37 \%$ & $3.24 \%$ \\
\hline MAE & RMSE & 1.0023 & 1.0005 & 1.0054 & 0.9998 \\
& MAE & 1.0006 & 0.9957 & 1.0011 & 1.0000 \\
& PC & $51.80 \%$ & $54.40 \%$ & $50.50 \%$ & $52.17 \%$ \\
& GAR & $2.05 \%$ & $5.91 \%$ & $-3.20 \%$ & $3.09 \%$ \\
\hline \multirow{2}{*}{ MSE } & RMSE & 1.0024 & 1.0005 & 1.0054 & 0.9998 \\
& MAE & 1.0006 & 0.9957 & 1.0011 & 1.0002 \\
& PC & $51.87 \%$ & $54.47 \%$ & $50.50 \%$ & $52.10 \%$ \\
& GAR & $2.00 \%$ & $6.52 \%$ & $-3.06 \%$ & $2.88 \%$ \\
\hline \multirow{2}{*}{ L } & RMSE & 1.0020 & 1.0006 & 1.0054 & 0.9996 \\
& MAE & 1.0005 & 0.9957 & 1.0012 & 1.0000 \\
& PC & $51.73 \%$ & $54.30 \%$ & $50.53 \%$ & $51.97 \%$ \\
& GAR & $1.83 \%$ & $5.89 \%$ & $-2.71 \%$ & $2.81 \%$
\end{tabular}

\begin{tabular}{ll|c|c|c|c|c} 
Weights & & EURUSD & EURCHF & EURJPY & EURGBP & Portfolio \\
\hline \multirow{2}{*}{ Equal } & RMSE & 0.9992 & 1.0015 & 1.0012 & 1.0007 & \\
& MAE & 0.9968 & 0.9960 & 0.9958 & 1.0034 & \\
& PC & $54.30 \%$ & $54.00 \%$ & $54.80 \%$ & $49.70 \%$ & $52.69 \%$ \\
& GAR & $8.00 \%$ & $3.01 \%$ & $6.56 \%$ & $-1.50 \%$ & $2.96 \%$ \\
\hline \multirow{2}{*}{ MAE } & RMSE & 0.9993 & 1.0015 & 1.0010 & 1.0007 & \\
& MAE & 0.9968 & 0.9960 & 0.9958 & 1.0034 & \\
& PC & $54.20 \%$ & $53.80 \%$ & $54.80 \%$ & $49.50 \%$ & $52.65 \%$ \\
& GAR & $7.63 \%$ & $2.82 \%$ & $6.56 \%$ & $-2.22 \%$ & $2.83 \%$ \\
\hline \multirow{2}{*}{ MSE } & RMSE & 0.9993 & 1.0015 & 1.0010 & 1.0007 & \\
& MAE & 0.9968 & 0.9960 & 0.9957 & 1.0034 & \\
& PC & $54.10 \%$ & $54.00 \%$ & $54.80 \%$ & $49.80 \%$ & $52.70 \%$ \\
& GAR & $7.25 \%$ & $2.90 \%$ & $6.56 \%$ & $-0.97 \%$ & $3.01 \%$ \\
\hline \multirow{2}{*}{ L } & RMSE & 0.9992 & 1.0015 & 1.0013 & 1.0007 & \\
& MAE & 0.9968 & 0.9960 & 0.9958 & 1.0029 & \\
& PC & $54.20 \%$ & $53.90 \%$ & $54.70 \%$ & $50.10 \%$ & $52.68 \%$ \\
& GAR & $8.46 \%$ & $2.96 \%$ & $5.70 \%$ & $-0.47 \%$ & $3.06 \%$
\end{tabular}

RWx denotes a rolling estimation window of $x$ observations. All forecasts are based on LAD-BI (KTC) estimates. See notes to Table 9.1 for further details on cell entries. 
Table 10.2

Combination of forecasts across estimation samples, RW100 + RW500 + RW1000

\begin{tabular}{ll|c|c|c|c} 
Weights & & GBPUSD & USDCHF & USDJPY & USDCAD \\
\hline Equal & RMSE & 1.0035 & 1.0023 & 1.0075 & 1.0020 \\
& MAE & 1.0006 & 0.9970 & 1.0020 & 1.0022 \\
& PC & $51.83 \%$ & $54.60 \%$ & $52.10 \%$ & $51.40 \%$ \\
& GAR & $1.43 \%$ & $4.76 \%$ & $0.49 \%$ & $2.54 \%$ \\
\hline \multirow{2}{*}{ MAE } & RMSE & 1.0036 & 1.0026 & 1.0083 & 1.0022 \\
& MAE & 1.0010 & 0.9972 & 1.0024 & 1.0024 \\
& PC & $51.87 \%$ & $54.60 \%$ & $51.80 \%$ & $51.40 \%$ \\
& GAR & $1.09 \%$ & $4.76 \%$ & $-0.75 \%$ & $2.61 \%$ \\
\hline \multirow{2}{*}{ MSE } & RMSE & 1.0036 & 1.0028 & 1.0092 & 1.0022 \\
& MAE & 1.0013 & 0.9976 & 1.0028 & 1.0024 \\
& PC & $52.03 \%$ & $54.57 \%$ & $51.70 \%$ & $51.50 \%$ \\
& GAR & $1.18 \%$ & $4.44 \%$ & $-0.85 \%$ & $3.37 \%$ \\
\hline \multirow{2}{*}{ L } & RMSE & 1.0032 & 1.0021 & 1.0068 & 1.0020 \\
& MAE & 1.0005 & 0.9965 & 1.0016 & 1.0019 \\
& PC & $52.00 \%$ & $54.67 \%$ & $52.27 \%$ & $51.50 \%$ \\
& GAR & $1.95 \%$ & $5.19 \%$ & $1.06 \%$ & $2.63 \%$
\end{tabular}

\begin{tabular}{ll|c|c|c|c|c} 
Weights & & EURUSD & EURCHF & EURJPY & EURGBP & Portfolio \\
\hline \multirow{2}{*}{ Equal } & RMSE & 1.0008 & 1.0009 & 1.0033 & 1.0033 & \\
& MAE & 0.9991 & 0.9944 & 0.9972 & 1.0057 & \\
& PC & $52.70 \%$ & $55.40 \%$ & $54.70 \%$ & $50.20 \%$ & $52.87 \%$ \\
& GAR & $5.05 \%$ & $4.59 \%$ & $7.72 \%$ & $-2.61 \%$ & $3.00 \%$ \\
\hline \multirow{2}{*}{ MAE } & RMSE & 1.0011 & 1.0018 & 1.0034 & 1.0029 & \\
& MAE & 0.9989 & 0.9956 & 0.9975 & 1.0053 & \\
& PC & $52.20 \%$ & $55.30 \%$ & $54.10 \%$ & $49.60 \%$ & $52.61 \%$ \\
& GAR & $3.87 \%$ & $4.18 \%$ & $5.70 \%$ & $-4.86 \%$ & $2.07 \%$ \\
\hline \multirow{2}{*}{ MSE } & RMSE & 1.0012 & 1.0024 & 1.0037 & 1.0029 & \\
& MAE & 0.9988 & 0.9960 & 0.9980 & 1.0053 & \\
& PC & $52.60 \%$ & $55.10 \%$ & $53.40 \%$ & $49.40 \%$ & $52.54 \%$ \\
& GAR & $4.63 \%$ & $3.81 \%$ & $3.22 \%$ & $-4.34 \%$ & $1.93 \%$ \\
\hline \multirow{2}{*}{ L } & RMSE & 1.0007 & 1.0000 & 1.0032 & 1.0033 & \\
& MAE & 0.9991 & 0.9936 & 0.9968 & 1.0057 & \\
& PC & $52.60 \%$ & $55.70 \%$ & $54.80 \%$ & $50.70 \%$ & $53.03 \%$ \\
& GAR & $5.70 \%$ & $4.66 \%$ & $8.98 \%$ & $-1.76 \%$ & $3.55 \%$
\end{tabular}

See notes to Table 10.1 for details on cell entries. 
Table 10.3

Combination of forecasts across estimation samples, OWMSE + OWMAE

\begin{tabular}{ll|c|c|c|c} 
Weights & & GBPUSD & USDCHF & USDJPY & USDCAD \\
\hline Equal & RMSE & 1.0023 & 1.0044 & 1.0067 & 1.0025 \\
& MAE & 1.0010 & 1.0015 & 1.0039 & 1.0026 \\
& PC & $52.53 \%$ & $53.67 \%$ & $52.47 \%$ & $50.50 \%$ \\
& GAR & $6.01 \%$ & $8.40 \%$ & $3.15 \%$ & $-1.06 \%$ \\
\hline \multirow{2}{*}{ MAE } & RMSE & 1.0023 & 1.0044 & 1.0067 & 1.0025 \\
& MAE & 1.0010 & 1.0015 & 1.0038 & 1.0026 \\
& PC & $52.50 \%$ & $53.63 \%$ & $52.47 \%$ & $50.53 \%$ \\
& GAR & $5.99 \%$ & $8.06 \%$ & $3.04 \%$ & $-1.03 \%$ \\
\hline \multirow{2}{*}{ MSE } & RMSE & 1.0022 & 1.0044 & 1.0066 & 1.0025 \\
& MAE & 1.0010 & 1.0015 & 1.0037 & 1.0026 \\
& PC & $52.50 \%$ & $53.63 \%$ & $52.53 \%$ & $50.50 \%$ \\
& GAR & $5.99 \%$ & $8.06 \%$ & $3.28 \%$ & $-1.06 \%$ \\
\hline \multirow{2}{*}{ L } & RMSE & 1.0023 & 1.0044 & 1.0068 & 1.0025 \\
& MAE & 1.0011 & 1.0015 & 1.0040 & 1.0026 \\
& PC & $52.57 \%$ & $53.73 \%$ & $52.53 \%$ & $50.50 \%$
\end{tabular}

\begin{tabular}{ll|c|c|c|c|c} 
Weights & & EURUSD & EURCHF & EURJPY & EURGBP & Portfolio \\
\hline \multirow{2}{*}{ Equal } & RMSE & 1.0025 & 1.0088 & 1.0129 & 1.0113 & \\
& MAE & 1.0061 & 1.0068 & 1.0094 & 1.0172 & \\
& PC & $52.20 \%$ & $53.70 \%$ & $53.00 \%$ & $50.50 \%$ & $52.32 \%$ \\
& GAR & $9.15 \%$ & $3.41 \%$ & $4.70 \%$ & $-2.37 \%$ & $3.93 \%$ \\
\hline \multirow{2}{*}{ MAE } & RMSE & 1.0025 & 1.0088 & 1.0129 & 1.0113 & \\
& MAE & 1.0061 & 1.0068 & 1.0094 & 1.0172 & \\
& PC & $52.20 \%$ & $53.70 \%$ & $53.00 \%$ & $50.50 \%$ & $52.32 \%$ \\
& GAR & $9.15 \%$ & $3.41 \%$ & $4.70 \%$ & $-2.37 \%$ & $3.87 \%$ \\
\hline \multirow{2}{*}{ MSE } & RMSE & 1.0025 & 1.0088 & 1.0128 & 1.0113 & \\
& MAE & 1.0059 & 1.0068 & 1.0094 & 1.0172 & \\
& PC & $52.30 \%$ & $53.70 \%$ & $53.00 \%$ & $50.50 \%$ & $52.33 \%$ \\
& GAR & $9.62 \%$ & $3.41 \%$ & $4.70 \%$ & $-2.37 \%$ & $3.95 \%$ \\
\hline \multirow{2}{*}{ L } & RMSE & 1.0025 & 1.0088 & 1.0130 & 1.0113 & \\
& MAE & 1.0062 & 1.0068 & 1.0095 & 1.0172 & \\
& PC & $52.10 \%$ & $53.70 \%$ & $53.10 \%$ & $50.50 \%$ & $52.34 \%$ \\
& GAR & $8.99 \%$ & $3.41 \%$ & $5.14 \%$ & $-2.37 \%$ & $4.08 \%$
\end{tabular}

OW $x$ denotes estimation samples optimized based on $x$. See notes to Table 10.1 for details on cell entries. 
Table 10.4

Combination of forecasts across estimation samples, RW1000 + EW

\begin{tabular}{ll|c|c|c|c} 
Weights & & GBPUSD & USDCHF & USDJPY & USDCAD \\
\hline Equal & RMSE & 1.0014 & 0.9989 & 1.0030 & 0.9989 \\
& MAE & 1.0002 & 0.9949 & 0.9997 & 0.9995 \\
& PC & $51.67 \%$ & $54.57 \%$ & $50.57 \%$ & $51.57 \%$ \\
& GAR & $1.48 \%$ & $8.05 \%$ & $-1.34 \%$ & $3.65 \%$ \\
\hline MAE & RMSE & 1.0013 & 0.9989 & 1.0030 & 0.9989 \\
& MAE & 1.0002 & 0.9949 & 0.9997 & 0.9995 \\
& PC & $51.47 \%$ & $54.57 \%$ & $50.63 \%$ & $51.57 \%$ \\
& GAR & $1.43 \%$ & $8.08 \%$ & $-1.16 \%$ & $3.81 \%$ \\
\hline \multirow{2}{*}{ MSE } & RMSE & 1.0012 & 0.9988 & 1.0030 & 0.9989 \\
& MAE & 1.0000 & 0.9949 & 0.9997 & 0.9995 \\
& PC & $51.37 \%$ & $54.67 \%$ & $50.97 \%$ & $51.87 \%$ \\
& GAR & $1.10 \%$ & $8.48 \%$ & $-0.34 \%$ & $4.44 \%$ \\
\hline L & RMSE & 1.0016 & 0.9989 & 1.0029 & 0.9991 \\
& MAE & 1.0003 & 0.9950 & 0.9996 & 0.9998 \\
& PC & $51.67 \%$ & $54.43 \%$ & $50.70 \%$ & $51.63 \%$
\end{tabular}

\begin{tabular}{ll|c|c|c|c|c} 
Weights & & EURUSD & EURCHF & EURJPY & EURGBP & Portfolio \\
\hline \multirow{2}{*}{ Equal } & RMSE & 0.9990 & 1.0003 & 1.0001 & 1.0033 & \\
& MAE & 0.9966 & 0.9964 & 0.9959 & 1.0057 & \\
& PC & $54.10 \%$ & $52.90 \%$ & $54.80 \%$ & $50.20 \%$ & $52.55 \%$ \\
& GAR & $8.96 \%$ & $1.38 \%$ & $7.37 \%$ & $-2.37 \%$ & $3.40 \%$ \\
\hline \multirow{2}{*}{ MAE } & RMSE & 0.9990 & 1.0003 & 1.0001 & 1.0033 & \\
& MAE & 0.9966 & 0.9964 & 0.9959 & 1.0057 & \\
& PC & $54.10 \%$ & $52.90 \%$ & $54.80 \%$ & $50.00 \%$ & $52.50 \%$ \\
& GAR & $8.96 \%$ & $1.54 \%$ & $7.37 \%$ & $-2.75 \%$ & $3.41 \%$ \\
\hline \multirow{2}{*}{ MSE } & RMSE & 0.9990 & 1.0003 & 1.0001 & 1.0033 & \\
& MAE & 0.9966 & 0.9964 & 0.9960 & 1.0057 & \\
& PC & $54.10 \%$ & $52.80 \%$ & $54.80 \%$ & $50.10 \%$ & $52.58 \%$ \\
& GAR & $8.96 \%$ & $1.51 \%$ & $7.37 \%$ & $-2.63 \%$ & $3.61 \%$ \\
\hline \multirow{2}{*}{ L } & RMSE & 0.9990 & 1.0003 & 1.0001 & 1.0029 & \\
& MAE & 0.9966 & 0.9964 & 0.9958 & 1.0057 & \\
& PC & $54.00 \%$ & $52.90 \%$ & $54.80 \%$ & $50.00 \%$ & $52.52 \%$ \\
& GAR & $8.76 \%$ & $1.38 \%$ & $7.37 \%$ & $-2.57 \%$ & $3.34 \%$
\end{tabular}

EW denotes an expanding estimation window. See notes to Table 10.1 for details on cell entries. 
Table 10.5

Combination of forecasts across estimation samples, EW + OWMAE

\begin{tabular}{ll|c|c|c|c} 
Weights & & GBPUSD & USDCHF & USDJPY & USDCAD \\
\hline Equal & RMSE & 1.0002 & 0.9993 & 1.0024 & 1.0009 \\
& MAE & 0.9982 & 0.9964 & 0.9988 & 1.0012 \\
& PC & $52.97 \%$ & $54.47 \%$ & $52.93 \%$ & $50.50 \%$ \\
& GAR & $6.54 \%$ & $9.69 \%$ & $5.53 \%$ & $-0.86 \%$ \\
\hline MAE & RMSE & 1.0000 & 0.9988 & 1.0020 & 1.0009 \\
& MAE & 0.9981 & 0.9954 & 0.9985 & 1.0012 \\
& PC & $53.30 \%$ & $54.47 \%$ & $52.30 \%$ & $50.50 \%$ \\
& GAR & $6.85 \%$ & $9.49 \%$ & $4.13 \%$ & $-0.86 \%$ \\
\hline \multirow{2}{*}{ MSE } & RMSE & 1.0000 & 0.9985 & 1.0018 & 1.0009 \\
& MAE & 0.9981 & 0.9947 & 0.9984 & 1.0010 \\
& PC & $52.90 \%$ & $54.60 \%$ & $52.17 \%$ & $50.63 \%$ \\
& GAR & $4.94 \%$ & $9.05 \%$ & $3.03 \%$ & $-0.60 \%$ \\
\hline \multirow{2}{*}{ L } & RMSE & 1.0006 & 0.9999 & 1.0030 & 1.0011 \\
& MAE & 0.9986 & 0.9974 & 0.9992 & 1.0014 \\
& PC & $52.93 \%$ & $54.23 \%$ & $52.67 \%$ & $50.47 \%$ \\
& GAR & $6.68 \%$ & $9.24 \%$ & $4.08 \%$ & $-0.91 \%$
\end{tabular}

\begin{tabular}{ll|c|c|c|c|c} 
Weights & & EURUSD & EURCHF & EURJPY & EURGBP & Portfolio \\
\hline Equal & RMSE & 0.9973 & 1.0021 & 1.0049 & 1.0058 & \\
& MAE & 0.9971 & 0.9980 & 1.0010 & 1.0077 & \\
& PC & $54.80 \%$ & $53.90 \%$ & $54.30 \%$ & $49.60 \%$ & $52.93 \%$ \\
& GAR & $15.33 \%$ & $2.64 \%$ & $6.63 \%$ & $-4.07 \%$ & $5.18 \%$ \\
\hline MAE & RMSE & 0.9973 & 1.0015 & 1.0037 & 1.0051 & \\
& MAE & 0.9970 & 0.9976 & 1.0001 & 1.0062 & \\
& PC & $54.80 \%$ & $54.00 \%$ & $54.10 \%$ & $49.80 \%$ & $52.91 \%$ \\
& GAR & $13.90 \%$ & $3.13 \%$ & $5.45 \%$ & $-4.19 \%$ & $4.74 \%$ \\
\hline \multirow{2}{*}{ MSE } & RMSE & 0.9973 & 1.0012 & 1.0027 & 1.0051 & \\
& MAE & 0.9968 & 0.9968 & 0.9992 & 1.0057 & \\
& PC & $55.10 \%$ & $53.80 \%$ & $54.60 \%$ & $49.90 \%$ & $52.96 \%$ \\
& GAR & $15.01 \%$ & $2.74 \%$ & $6.52 \%$ & $-3.93 \%$ & $4.59 \%$ \\
\hline L & RMSE & 0.9974 & 1.0027 & 1.0063 & 1.0066 & \\
& MAE & 0.9975 & 0.9988 & 1.0022 & 1.0086 & \\
& PC & $54.90 \%$ & $54.40 \%$ & $54.20 \%$ & $49.40 \%$ & $52.90 \%$ \\
& GAR & $15.88 \%$ & $2.42 \%$ & $6.72 \%$ & $-4.52 \%$ & $4.95 \%$
\end{tabular}

EW denotes an expanding estimation window and OWx denotes estimation samples optimized based on $x$. See notes to Table 10.1 for details on cell entries. 
Table 10.6

Combination of forecasts across estimation samples, RW1000 + EW + OWMAE

\begin{tabular}{ll|c|c|c|c} 
Weights & & GBPUSD & USDCHF & USDJPY & USDCAD \\
\hline Equal & RMSE & 1.0006 & 0.9991 & 1.0025 & 0.9996 \\
& MAE & 0.9987 & 0.9953 & 0.9989 & 1.0000 \\
& PC & $53.07 \%$ & $54.37 \%$ & $52.23 \%$ & $51.30 \%$ \\
& GAR & $5.09 \%$ & $9.38 \%$ & $3.05 \%$ & $2.40 \%$ \\
\hline \multirow{2}{*}{ MAE } & RMSE & 1.0005 & 0.9989 & 1.0024 & 0.9993 \\
& MAE & 0.9989 & 0.9950 & 0.9990 & 0.9998 \\
& PC & $52.87 \%$ & $54.17 \%$ & $52.17 \%$ & $51.40 \%$ \\
& GAR & $4.35 \%$ & $8.15 \%$ & $2.47 \%$ & $2.87 \%$ \\
\hline \multirow{2}{*}{ MSE } & RMSE & 1.0006 & 0.9987 & 1.0025 & 0.9993 \\
& MAE & 0.9990 & 0.9946 & 0.9991 & 0.9998 \\
& PC & $52.67 \%$ & $54.37 \%$ & $51.90 \%$ & $51.50 \%$ \\
& GAR & $3.08 \%$ & $7.94 \%$ & $1.47 \%$ & $3.48 \%$ \\
\hline \multirow{2}{*}{ L } & RMSE & 1.0007 & 0.9994 & 1.0028 & 0.9998 \\
& MAE & 0.9987 & 0.9960 & 0.9990 & 1.0002 \\
& PC & $53.07 \%$ & $54.30 \%$ & $52.60 \%$ & $50.93 \%$ \\
& GAR & $6.29 \%$ & $9.30 \%$ & $3.46 \%$ & $1.48 \%$
\end{tabular}

\begin{tabular}{ll|c|c|c|c|c} 
Weights & & EURUSD & EURCHF & EURJPY & EURGBP & Portfolio \\
\hline \multirow{2}{*}{ Equal } & RMSE & 0.9974 & 1.0009 & 1.0031 & 1.0040 & \\
& MAE & 0.9964 & 0.9964 & 0.9988 & 1.0057 & \\
& PC & $55.50 \%$ & $54.50 \%$ & $54.90 \%$ & $49.50 \%$ & $53.17 \%$ \\
& GAR & $14.84 \%$ & $3.51 \%$ & $6.98 \%$ & $-5.05 \%$ & $5.02 \%$ \\
\hline \multirow{2}{*}{ MAE } & RMSE & 0.9974 & 1.0006 & 1.0024 & 1.0037 & \\
& MAE & 0.9964 & 0.9964 & 0.9982 & 1.0053 & \\
& PC & $56.10 \%$ & $54.60 \%$ & $54.90 \%$ & $49.30 \%$ & $53.19 \%$ \\
& GAR & $17.09 \%$ & $3.83 \%$ & $7.20 \%$ & $-5.53 \%$ & $5.05 \%$ \\
\hline \multirow{2}{*}{ MSE } & RMSE & 0.9974 & 1.0006 & 1.0018 & 1.0037 & \\
& MAE & 0.9964 & 0.9964 & 0.9976 & 1.0053 & \\
& PC & $56.20 \%$ & $53.90 \%$ & $55.00 \%$ & $48.90 \%$ & $53.05 \%$ \\
& GAR & $18.05 \%$ & $3.00 \%$ & $7.08 \%$ & $-5.46 \%$ & $4.83 \%$ \\
\hline \multirow{2}{*}{ L } & RMSE & 0.9974 & 1.0012 & 1.0040 & 1.0044 & \\
& MAE & 0.9966 & 0.9968 & 0.9995 & 1.0067 & \\
& PC & $55.30 \%$ & $54.40 \%$ & $55.00 \%$ & $49.80 \%$ & $53.18 \%$ \\
& GAR & $14.03 \%$ & $3.23 \%$ & $8.30 \%$ & $-3.66 \%$ & $5.30 \%$
\end{tabular}

EW denotes an expanding estimation window, and OW $x$ denotes estimation samples optimized based on $x$. See notes to Table 10.1 for details on cell entries. 